\title{
Identification of PKCa-dependent phosphoproteins in mouse retina
}

Short title: PKCa-dependent phosphoproteins in mouse retina

Colin M. Wakeham ${ }^{1}$, Phillip A. Wilmarth², Jennifer M. Cunliffe ${ }^{2}$, John E. Klimek², Gaoying Ren, Larry L. David ${ }^{2,3}$, Catherine W. Morgans ${ }^{1}$

${ }^{1}$ Department of Physiology and Pharmacology, Oregon Health and Science University, Portland, OR 97239, USA

${ }^{2}$ Proteomics Shared Resource, Oregon Health and Science University, Portland, OR 97239, USA

${ }^{3}$ Department of Biochemistry and Molecular Biology, Oregon Health and Science University, Portland, OR 97239, USA

Corresponding author: Catherine Morgans

Department of Physiology \& Pharmacology, L-334

Oregon Health \& Science University

3181 SW Sam Jackson Park Rd

Portland, OR 97239

Email: morgansc@ohsu.edu 
Acknowledgments: The authors would like to thank Tammie L. Haley for producing mouse retina sections for immunofluorescence experiments. This work was supported by the National Institutes of Health grants R01EY022369 and 5P30EY010572 and an OHSU University Shared Resources Core Pilot Fund grant.

Conflict of interest statement: The authors declare no conflicts of interest.

Author contributions: study design: CMW, LLD, CWM; experimentation: CMW, JMC, PAW, GR, CWM; PAW Pipeline script development: PAW; data analysis: CMW, PAW, JEK, LLD, CWM; figure and manuscript preparation: CMW, JMC, PAW, JEK, LLD, CWM; editing and review: CMW, JMC, PAW, GR, JEK, LLD, CWM; repository preparation: CMW, PAW; supplemental files preparation: CMW, PAW

Abbreviations: BORG4: Binder of Rho GTPase 4; DAG: diacylglycerol; ERG: electroretinogram; DE: differential expression; FC: fold change; FDR: false discovery rate; GCL: ganglion cell layer; INL: inner nuclear layer; IPL: inner plexiform layer; KO: knockout; LC-MS/MS: liquid chromatography tandem mass spectroscopy; NHERF1: $\mathrm{Na}^{+} / \mathrm{H}^{+}$Exchange Regulatory Factor 1; OPL: outer plexiform layer; PKCa/Prkca: Protein Kinase C-alpha; PMA: phorbol 12-myristate 13acetate; PSM: peptide spectrum match; RBC: rod bipolar cell; RPE: retinal pigment epithelium; TMT: tandem mass tag; TPBG: Trophoblast Glycoprotein; TRPM1: Transient Receptor Potential cation channel subfamily M member 1

Keywords: retina, rod bipolar cell, protein kinase C-alpha, quantitative phosphoproteomics, TPBG, BORG4 


\section{Abstract}

Adjusting to a wide range of light intensities is an essential feature of retinal rod bipolar cell (RBC) function. While persuasive evidence suggests this modulation involves phosphorylation by protein kinase C-alpha (PKCa), the targets of PKCa phosphorylation in the retina have not been identified. PKCa activity and phosphorylation in RBCs was examined by immunofluorescence confocal microscopy using a conformation-specific PKCa antibody and antibodies to phosphorylated PKC motifs. PKCa activity was dependent on light and expression of TRPM1, and RBC dendrites were the primary sites of light-dependent phosphorylation. PKCadependent retinal phosphoproteins were identified using a phosphoproteomics approach to compare total protein and phosphopeptide abundance between phorbol ester-treated wild type and PKCa knockout (PKCa-KO) mouse retinas. Phosphopeptide mass spectrometry identified over 1100 phosphopeptides in mouse retina, with 12 displaying significantly greater phosphorylation in WT compared to PKCa-KO samples. The differentially phosphorylated proteins fall into the following functional groups: cytoskeleton/trafficking (4 proteins), ECM/adhesion (2 proteins), signaling (2 proteins), transcriptional regulation (3 proteins), and homeostasis/metabolism (1 protein). Two strongly differentially expressed phosphoproteins, BORG4 and TPBG, were localized to the synaptic layers of the retina, and may play a role in PKCa-dependent modulation of RBC physiology. Data are available via ProteomeXchange with identifier PXD012906.

\section{Significance}

Retinal rod bipolar cells (RBCs), the second-order neurons of the mammalian rod visual pathway, are able to modulate their sensitivity to remain functional across a wide range of light intensities, from starlight to daylight. Evidence suggests that this modulation requires the serine/threonine kinase, PKCa, though the specific mechanism by which PKCa modulates RBC physiology is unknown. This study examined PKCa phosophorylation patterns in mouse rod 
bipolar cells and then used a phosphoproteomics approach to identify PKCa-dependent phosphoproteins in the mouse retina. A small number of retinal proteins showed significant PKCadependent phosphorylation, including BORG4 and TPBG, suggesting a potential contribution to PKCa-dependent modulation of RBC physiology.

\section{Highlights}

- PKCa is a major source of phosphorylation in retinal RBC dendrites and its activity in RBCs is light dependent.

- Proteins showing differential phosphorylation between phorbol ester-treated wild type and $\mathrm{PKC \alpha -KO}$ retinas belong to the following major functional groups: cytoskeleton/trafficking (4 proteins), ECM/adhesion (2 proteins), signaling (2 proteins), transcriptional regulation (3 proteins), and homeostasis/metabolism (1 protein).

- $\quad$ The PKCa-dependent phosphoproteins, BORG4 and TPBG, are present in the synaptic layers of the retina and may be involved in PKCa-dependent modulation of RBC physiology.

\section{Introduction}

Rod bipolar cells (RBCs) are key retinal interneurons in the rod visual pathway that receive light-driven synaptic input from rod photoreceptors and drive retinal output via synapses onto All amacrine cells. RBCs serve different visual functions depending on luminance conditions. When dark adapted, they are able to transmit single photon responses [1,2], allowing for useful vision in starlight. RBCs are also able to transmit contrast changes against dim background light $[3,4]$, and have recently been shown to influence vision in daylight [5]. Little is known about how RBCs adjust their sensitivity and gain to transition between these modes, but compelling evidence suggests phosphorylation by protein kinase C-alpha (PKCa) may play a role. 
$\mathrm{PKCa}$ is a serine/threonine protein kinase that undergoes calcium-dependent translocation from the cytosol to the plasma membrane, where it is activated upon binding to diacylglycerol (DAG). It is a powerful modulator of signal transduction pathways and is so abundant in RBCs that it is used as a cell marker to identify RBCs with retinal immunofluorescence [6-8]. The light response of RBCs is reflected in the b-wave of the dark-adapted electroretinogram (ERG), and comparison of ERGs from wild type (WT) and PKCa knockout (PKCa-KO) mice reveal that genetic deletion of PKCa results in increases in both amplitude and duration of the scotopic b-wave $[9,10]$. This effect is particularly dramatic at brighter light intensities, suggesting that PKCa modulates the light response in an intensity-dependent manner. If the effect of PKCa on the light response is due to its kinase activity, then RBC proteins phosphorylated by PKC $\alpha$ are also likely to be involved in the modulation of RBC activity.

We used a multiplexed tandem mass tag (TMT; Thompson et al., 2003) mass spectrometry-based phosphoproteomics approach to identify proteins that were differentially phosphorylated between WT and PKCa-KO retinas in order to gain insight into the biochemical mechanisms and pathways that mediate the effect of PKCa in RBCs. Phosphopeptide abundance is expected to be dynamic, so TMT acquisition methods that have improved accuracy and wider dynamic ranges are necessary [12]. The larger number of replicates available with high-resolution instruments and TMT tags require improved data normalization and statistical testing methods, and we have successfully applied analysis techniques developed for large-scale protein expression studies [13] to phosphopeptide abundance data. Since PKCa-dependent changes in protein phosphorylation may be due to changes in total protein abundance, we also identified proteins that were differentially expressed between WT and PKCa-KO retinas.

\section{Experimental Procedures}

Experimental design and statistical rationale 
For quantification of immunofluorescence (Figure 2C), we used retina sections from 4 WT and 3 TRPM-1-KO mice, each with 4 technical replicates. For the multiplexed TMT mass spectroscopy experiments, we used 4 WT and 5 PKC $\alpha-K O$ mice. The $5 \times 5$ study design (one WT sample was lost) was the maximum number of samples accommodated by 10 -plex TMT. For both total protein and phosphopeptide experiments, all nine samples were pooled after isobaric labeling and run simultaneously to reduce variability between samples. The statistical tests used are described within the Statistical analysis of differential expression section.

\section{Animals}

All studies were approved by the Institutional Animal Care and Use Committee at Oregon Health and Science University. Wild type mice used were C57BL/6J (Jackson Laboratory; Bar Harbor, ME, USA; Cat\# 000664). The PKC $\alpha-K O$ mouse strain was B6;129-Pkrca ${ }^{\mathrm{tm} 1 \mathrm{Jmk} / J}$ (Jackson Laboratory; Cat\# 009068; [10]). The TRPM1-KO mouse strain was TRPM1 ${ }^{\text {tm1Lex }}$ (Texas A\&M Institute of Genomic Medicine; College Station, TX, USA; [14]).

PKC $\alpha$ activation by PMA treatment for immunoblotting or immunofluorescence

Freshly dissected retinas from WT and PKCa-KO mice were incubated at $37^{\circ} \mathrm{C}$ in $1 \mu \mathrm{M}$ phorbol 12-myristate 13-acetate (PMA; Sigma-Aldrich; St. Louis, MO, USA; Cał\# P8139) diluted in bicarbonate buffered Ames medium (US Biological Life Sciences; Salem, MA, USA; Cat\# A1372-25) for $0 \mathrm{~min}, 15 \mathrm{~min}, 30 \mathrm{~min}$, or $60 \mathrm{~min}$. The retinas were then washed three times with Ames medium before being either fixed for cryo-sectioning or processed for western blotting. 


\section{Immunoblotting}

Retinas were suspended in chilled lysis buffer (50 mM Tris pH 7.4, $150 \mathrm{mM} \mathrm{NaCl}, 1 \mathrm{mM}$ EDTA, $1 \%$ Triton $\mathrm{X}-100,1 \%$ deoxycholate, $0.1 \%$ SDS) with $1 \mathrm{X}$ protease/phosphatase inhibitor cocktail (Cell Signaling Technology; Danvers, MA, USA; Cat\# 5872). The retinas were then homogenized and incubated on ice for 1 hour. Lysates were centrifuged for $15 \mathrm{~min}$ at 28,000 x g and $4{ }^{\circ} \mathrm{C}$, and the pellets discarded. Lysates were stored at $-20^{\circ} \mathrm{C}$. Equal quantities of WT and PKCa-KO retinal proteins were subjected to electrophoresis on precast NuPAGE 1mm 4-12\% Bis-Tris gels (Thermo Scientific; Waltham, MA, USA; Cat\# NP0322BOX). The separated proteins were transferred to polyvinylidene difluoride (PVDF) membranes and then blocked with Odyssey Blocking Buffer (LI-COR Biosciences; Lincoln, NE, USA; Cat\# 927-50003) for $1 \mathrm{hr}$. The blots were probed with primary antibody for $1 \mathrm{hr}$ at room temperature. The membranes were washed $3 \times 5$ min with TBST (Tris-buffered saline with $0.1 \%$ Tween-20) and then incubated for $1 \mathrm{hr}$ at room temperature with secondary antibody and washed $3 \times 5$ min with TBST. Immunoreactive bands were visualized with a LI-COR Odyssey CLx Imaging System at 700 or $800 \mathrm{~nm}$.

Primary antibodies used for immunoblotting were rabbit anti-PKC motif phosphoserine [(R/KXpSX(R/K)] MultiMab mAb mix (1:250; Cell Signaling Technology; Cat\# 6967), rabbit antiBorg4 (1:200; Bethyl Laboratories; Montgomery, TX, USA; Cat\# A302-379A), rabbit anti-5T4 (1:5000; Abcam; Cambridge, UK; Cat\# ab129058), rabbit anti-NHERF1 (1:5000; Abcam; Cambridge, UK; Cat\# ab3452). Secondary antibodies used were 680RD anti-rabbit (1:10,000; LICOR Biosciences; Cat\# 925-68071) and 800CW anti-mouse (1:10,000; LI-COR Biosciences; Cat\# 925-32212).

\section{Retinal immunofluorescence}

Mouse eyecups were prepared by cutting the sclera behind the ora serrata and removing the cornea and lens. Eyecups were fixed for $30 \mathrm{~min}$ by immersion in $4 \%$ paraformaldehyde, 
followed by washing in PBS. The fixed tissue was cryoprotected via sequential immersion in 10, 20, and $30 \%$ sucrose, and then embedded in Tissue-Tek O.C.T. Compound (Sakura Finetek; Torrance, CA, USA; Cat\# 4583) and frozen. Sections were cut at $25 \mu \mathrm{m}$ thickness on a cryostat and mounted onto glass slides, then air dried and stored at $-20^{\circ} \mathrm{C}$. Thawed retina sections were blocked at room temperature for $1 \mathrm{hr}$ in Antibody Incubation Solution (AIS: 3\% normal horse serum, $0.5 \%$ Triton $\mathrm{X}-100,0.025 \% \mathrm{NaN}_{3}$ in PBS). The sections were then incubated in primary antibody diluted in AIS for $1 \mathrm{hr}$ at room temperature. After washing with $3 \mathrm{x}$ with PBS, the sections were incubated for $1 \mathrm{hr}$ at room temperature in secondary antibody diluted 1:1000 in AIS. The slides were washed again 3x in PBS and coverslips applied with Lerner Aqua-Mount (Thermo Scientific; Cat\# 13800).

Primary antibodies used for immunofluorescence were the same as used for immunoblotting unless indicated: rabbit anti-PKC motif phosphoserine [(R/KXpSX(R/K)] MultiMab mAb mix; 1:250), rabbit anti-PKCa (1:5000; Sigma-Aldrich; Cat\# P4334), mouse anti-PKCa clone MC5 (1:5000; Sigma-Aldrich; Cat\# P5704), rabbit anti-Borg4 (1:500), rabbit anti-5T4 (1:500;), rabbit anti-NHERF1 (1:100). Secondary antibodies used were anti-rabbit-AF488 (1:1000; Jackson ImmunoResearch Labs; West Grove, PA, USA; Cat\# 11-545-144) and anti-mouse-Cy3 (1:1000 Jackson ImmunoResearch Labs; Cat\# 115-165-003).

\section{Scanning confocal imaging}

Confocal immunofluorescence images were taken with a Leica TCS SP8 X confocal microscope (Leica; Wetzlar, Germany) using a Leica HC PL APO CS2 63x/1.40 oil immersion objective (Leica; Cat\#15506350) and Leica HyD hybrid detectors, or with an Olympus Fluoview 1000 microscope (Olympus; Tokyo, Japan) using a 60x/1.42 oil immersion objective. Laser lines used were AF488 (499 nm), and Cy3 (554 nm). Detection windows used were AF488 (509- 
544nm) and Cy3 (564-758) nm. Brightness and contrast were adjusted using Olympus Fluoview software, Leica LAS X software, or ImageJ $[15,16]$.

\section{Preparation of retinas for TMT analysis}

To maximize the difference between groups, retinas from wild type $(n=4)$ and PKCa-KO $(n=5)$ mice were extracted and treated for 1 hour at $37^{\circ} \mathrm{C}$ with $1 \mu \mathrm{M}$ PMA diluted in bicarbonate buffered Ames medium. Following treatment, retinas were washed three times with Ames medium before being placed in chilled lysis buffer (50 mM HEPES, pH 8.5, 8 M urea, 1 mM NaF, 1 mM sodium orthovanadate, $10 \mathrm{mM}$ sodium pyrophosphate, $1 \mathrm{mM}$ beta-glycerophosphate), and lysed by probe sonication (Sonic Dismembrator 60; Thermo Scientific) $3 \times 15$ seconds at a setting of 4 with cooling on ice for 30 seconds between treatments. Protein concentrations were determined using the Pierce BCA Protein Assay Kit (Thermo Scientific; Cat\# 223227) and approximately $500 \mu \mathrm{g}$ of protein in $250 \mu \mathrm{L}$ of lysis buffer was used for further processing. Protein disulfides were reduced with $5 \mu \mathrm{L}$ of $1.25 \mathrm{M}$ dithiothreitol at $55^{\circ} \mathrm{C}$ for $30 \mathrm{~min}$, then alkylated by adding $22 \mu \mathrm{L}$ of 1 $\mathrm{M}$ iodoacetamide and incubation at room temperature in the dark for $15 \mathrm{~min}$, followed by an additional $5 \mu \mathrm{L}$ of $1.25 \mathrm{M}$ dithiothreitol. Water was added to dilute the urea concentration to $2 \mathrm{M}$. Sequence grade modified trypsin (Promega; Madison, WI, USA; Cat\# V5111) was added at a 25:1 protein:trypsin ratio and samples were incubated overnight at $37^{\circ} \mathrm{C}$ before being acidified with trifluoroacetic acid (TFA) to a final concentration of $1 \%$. Remaining particulates were removed by centrifugation at $16,000 \times \mathrm{g}$ for $10 \mathrm{~min}$.

Peptides were purified by solid phase extraction using 1 cc (50 mg) Waters Sep-Pak Vac tC18 cartridges (Waters Corporation; Milford, MA, USA; Cat\# WAT054960). Briefly, the cartridges were conditioned twice with $1 \mathrm{~mL}$ acetonitrile (ACN) and twice with $300 \mu \mathrm{L}$ of $50 \%$ ACN/0.5\% acetic acid, then equilibrated twice with $1 \mathrm{~mL} 0.1 \%$ TFA. The samples were loaded and passed through the bed, then were washed twice with $1 \mathrm{~mL} 0.1 \%$ TFA followed by $200 \mu \mathrm{L}$ of $0.5 \%$ acetic 
acid. Finally, the samples were eluted twice with $500 \mu \mathrm{L}$ of $50 \%$ ACN $/ 0.5 \%$ acetic acid. Peptide concentrations were determined using the Pierce Quantitative Colorimetric Peptide Assay (Thermo Scientific; Cat\# 23275). Approximately $300 \mu \mathrm{g}$ of peptide was recovered from each digest. Fifteen $\mu \mathrm{g}$ of each sample was reserved for TMT analysis of total protein abundance, and the remainder was used for the phosphopeptide enrichment experiment.

\section{Phosphopeptide enrichment}

Phosphopeptides were enriched following previously described methods $[17,18]$ with small modifications. Titanosphere $\mathrm{TiO}_{2} 5 \mu \mathrm{m}$ beads (GL Biosciences; Tokyo, Japan; Cat\# 502075000 ) were washed three times in $2 \mathrm{M}$ lactic acid/50\% $\mathrm{ACN}$, then resuspended in the same solution at $24 \mathrm{mg} / \mathrm{mL}$. Approximately $285 \mu \mathrm{g}$ of dried peptide for each sample was resuspended in $950 \mu \mathrm{L}$ of $2 \mathrm{M}$ lactic acid/50\% ACN and $100 \mu \mathrm{L}(2.4 \mathrm{mg})$ of the bead suspension was added to each sample, ensuring an 8:1 ratio of beads:peptide. The bead:peptide mixture was rotated at room temperature for 1 hour, then washed twice with $500 \mu \mathrm{L} 2 \mathrm{M}$ lactic acid $/ 50 \%$ ACN, $0.1 \%$ TFA $/ 50 \%$ ACN, then $0.1 \% \mathrm{TFA} / 25 \% \mathrm{ACN}$. The enriched phosphopeptides were eluted from the beads by vortexing in $100 \mu \mathrm{L}$ of $50 \mathrm{mM} \mathrm{K}_{2} \mathrm{HPO}_{4}$ at $\mathrm{pH} 10$ for $5 \mathrm{~min}$. The elution step was repeated once and the $200 \mu \mathrm{L}$ of eluate was dried by vacuum centrifugation.

The enriched phosphopeptides were then purified by solid phase extraction using UltraMicro Spin columns (The Nest Group Inc.; Southborough, MA, USA). The dried phosphopeptides were resuspended in $60 \mu \mathrm{L} 1 \%$ TFA and the $\mathrm{pH}$ was tested to ensure the samples were acidic. The columns were conditioned three times with $100 \mu \mathrm{L} 80 \%$ ACN/0.1\% TFA and equilibrated three times with $50 \mu \mathrm{L}$ of $0.1 \%$ TFA. The samples were loaded and passed through the columns three times, washed three times with $25 \mu \mathrm{L}$ of $0.1 \%$ TFA, eluted three times with $50 \mu \mathrm{L} 80 \% \mathrm{ACN} / 0.1 \%$ formic acid, and dried by vacuum centrifugation before TMT labeling. 
TMT labeling and mass spectrometric analysis

In preparation for TMT labeling, nine dried unfractionated peptide samples (4 WT and 5 KO) and nine phosphopeptide enriched samples (4 WT and $5 \mathrm{KO}$ ) were dissolved in $25 \mu \mathrm{L}$ of 100 mM triethylammonium bicarbonate buffer, and TMT 10-plex reagents (Thermo Scientific; Cat\# 90110) were dissolved at a concentration of $15 \mu \mathrm{g} / \mu \mathrm{L}$ in anhydrous ACN. Each of the samples was then labeled by adding $12 \mu \mathrm{L}(180 \mu \mathrm{g})$ of an individual TMT reagent, followed by shaking at room temperature for $1 \mathrm{hr}$. Two $\mu \mathrm{L}$ of each of the nine labeled samples in each group were pooled, and $2 \mu \mathrm{L}$ of $5 \%$ hydroxylamine was added. The samples were incubated for $15 \mathrm{~min}$, dried by vacuum centrifugation, dissolved in $21 \mu \mathrm{L}$ of $5 \%$ formic acid, and peptides were analyzed by a single 2-hour LC-MS/MS method using an Orbitrap Fusion as described below. The run was performed to normalize the total reporter ion intensity of each multiplexed sample and to check labeling efficiency. After the normalization and efficiency run, the remaining unmixed samples were then quenched by the addition of $2 \mu \mathrm{L} 5 \%$ hydroxylamine, then combined in adjusted volumes to yield equal summed reporter ion intensities during the subsequent two-dimensional LC/MS.

Following volume-based normalization, the combined samples were dried by vacuum centrifugation, and TMT-labeled samples were reconstituted in $20 \mu \mathrm{L}$ water. The reconstituted peptides were separated by two-dimensional nano reverse-phase liquid chromatography (Dionex NCS-3500 UltiMate RSLCnano UPLC) EasySpray NanoSource (Thermo Scientific), ionized using an EasySpray NanoSource (Thermo Scientific), and SPS MS3 data acquired with an Orbitrap Fusion Tribrid mass spectrometer (Thermo Scientific). The liquid chromatography details and mass spectrometer settings were as previously described [13] with the modification that nonenriched peptides were eluted from the first dimension high $\mathrm{pH}$ column using sequential injections of $20 \mu \mathrm{L}$ volumes of $14,17,20,21,22,23,24,25,26,27,28,29,30,35,40,50$, and $90 \%$ ACN in $10 \mathrm{mM}$ ammonium formate, $\mathrm{pH} \mathrm{9,} \mathrm{and} \mathrm{enriched} \mathrm{phosphopeptides} \mathrm{were} \mathrm{eluted} \mathrm{by} \mathrm{sequential} 20$ 
$\mu \mathrm{L}$ injections of $4,6,8,10,12,18,20,22,25,30$, and $60 \% \mathrm{ACN}$ in $10 \mathrm{mM}$ ammonium formate, $\mathrm{pH} 9$.

\section{TMT data analysis}

The binary instrument files were processed with Proteome Discoverer version 1.4 (Thermo Scientific) to extract fragment ion spectra, precursor information ( $\mathrm{m} / \mathrm{z}$ values and peptide charge state), and TMT reporter ion peak intensities. The fragment ion spectra were searched against a canonical Uniprot Swiss-Prot mouse protein database (downloaded 07/07/2016 from https://www.uniprot.org) with 16,794 entries. There were 179 common contaminant entries appended for a total of 16,973 sequences.

The SEQUEST [19] search engine was used with a parent ion mass tolerance of 1.25 Da and fragment ion tolerance of $1.0005 \mathrm{Da}$. Trypsin cleavage with up to two missed cleavages was specified. A static modification of +57.0215 Da was applied to all cysteine residues and a variable modification of +15.9949 was applied to methionine. Confident peptide-to-spectrum matches (PSMs) were obtained using the percolator [20] node, and only peptides with q values $<0.05$ were accepted. Parsimonious protein inference was used in Proteome Discoverer to produce final protein lists, and the results were exported to tab-delimited files for post processing using inhouse Python scripts (https://www.github.com/pwilmart/PAW_pipeline.git). For the protein expression analysis of the total protein preparations, the reporter ion intensities from all unique (matching to just one protein) peptides were summed into protein reporter ion intensities. Any contaminant proteins were excluded from further analysis. Reporter ion data from PSMs where the trimmed average reporter ion intensity did not exceed 500 were excluded. Any final protein reporter ion sums of zero were replaced with a value of 150 (the smallest non-zero reporter ion intensities observed were approximately 350) to avoid mathematical errors during visualizations and statistical testing. 
The data from the phosphopeptide enrichment experiment was searched with additional variable modifications of +79.9799 on serine, threonine, or tyrosine residues, and only peptides with $q<0.01$ were accepted. The phosphorylation site localization node phosphoRS [21] was configured after the search node in Proteome Discoverer. Phosphorylation enrichment experiments are peptide centric, so data aggregation was done differently than for protein expression. Peptide sequences were aggregated by summing reporter ion intensities within each channel to reduce variance and increase statistical power. All PSMs assigned to the same base peptide sequence were aggregated in the same modification state, which was determined by integral peptide $\mathrm{MH}+$ mass (the peptide in a $1+$ charge state). Localization information from phosphoRS was simplified to the same number of top probabilities as the number of phosphorylation modifications present in the peptides. The reporter ion intensities from the combined reports were used for differential expression (DE) testing as described below. Any contaminant protein matches were excluded from further analysis. Minimum intensity filtering and zero replacement was done similarly to the total protein analysis.

\section{Statistical analysis of differential expression}

For immunofluorescence quantification, data are represented in text as the mean \pm SEM and $\mathrm{p}$-values were calculated using an unpaired t-test.

For TMT data, the table of non-contaminant reporter ion intensities for proteins or for aggregated phosphopeptides were exported to tab-delimited files and imported into $\mathrm{R}$ (https://www.r-project.org) for statistical analysis using the edgeR [22-24] Bioconductor package. Normalization was done using a "library size" factor and the trimmed mean of M-values normalization [25] function in edgeR was used to correct for sample loading and compositional bias. DE testing was performed pairwise using the exact test with Benjamini-Hochberg multiple testing corrections [26]. The results from edgeR analyses were exported and added to the 
combined data summaries. Annotation information was added from Uniprot using a script available at https://github.com/pwilmart/annotations.

\section{Data availability}

The mass spectrometry proteomics data have been deposited to the ProteomeXchange Consortium via the PRIDE partner repository [27], with the dataset identifier PXD012906.

\section{Results}

\section{RBC dendrites are major sites of light-dependent PKCa phosphorylation}

To visualize sites of PKCa phosphorylation in the retina, we used a monoclonal antibody mix that binds to canonical PKC substrate motifs containing a phosphorylated serine (PKC motif p-serine). Anti-PKC motif phosphoserine immunofluorescence between wild type and PKCa-KO retina sections indicates that $\mathrm{RBC}$ dendrites are the main sites of PKCa phosphorylation in the mouse retina (Figure $1 \mathrm{~A}$ and $\mathrm{B}$ ). The small immunofluorescent puncta in the outer plexiform layer (OPL) are presumptive RBC dendrites, whereas the larger patches of phosphoserine immunofluorescence (arrows) are associated with cone pedicles. The small immunofluorescent puncta are visible throughout the wild type OPL but are greatly reduced in intensity in the PKCaKO retina, while immunofluorescence corresponding to cone pedicles is unchanged. These results suggest that PKCa phosphorylates targets in RBC dendrites of wild type adult mice, and that a different PKC isoform phosphorylates targets associated with cone pedicles.

To examine whether PKCa activity is light-dependent, we used a conformation-dependent monoclonal PKCa antibody which binds an epitope in the hinge region of PKCa that is inaccessible in the inactive state [28]. Retina sections from light-adapted and dark-adapted mice were double-labeled with the conformation-specific antibody (anti-PKCa-A) and a nonconformation-specific PKCa antibody (anti-PKCa-B; Figure 2). Both antibodies strongly label RBC 
cell bodies and dendrites in sections from light-adapted retina, with co-localization of the two secondary antibodies appearing white (Figure 2A, left). By contrast, only anti-PKCa-B labels RBCs in sections from dark adapted retina (Figure 2A, right). These results suggest that PKCa is active in RBC dendrites in the light-adapted state. This was supported by labeling of light- and dark-adapted retina sections for phosphorylated PKC motifs. The anti-PKC motif phosphoserine antibody labeled puncta in the OPL of light-adapted retina (Figure 2B, left), similar to the wild type immunofluorescence seen in Figure 1. Anti-PKC motif phosphoserine immunofluorescence was absent in the OPL of the dark-adapted retina (Figure 2B, right). Together, the results from Figures 1 and 2 indicate that PKCa is active in RBC dendrites in the light-adapted state.

Conventional PKC isoforms, including PKCa, require calcium for activation [29]. In RBC dendrites, a likely source of calcium is the Transient Receptor Potential cation channel subfamily M 1 (TRPM1) cation channel, which mediates an influx of sodium and calcium to generate the RBC light response [14,30,31]. The dependence of PKC $\alpha$ activation on TRPM1 was assessed by double-labeling retina sections from wild type and TRPM1 knockout (TRPM1-KO) mice with antiPKCa-A and anti-PKCa-B to detect active and total PKCa, respectively, in RBC cell bodies and dendrites (Figure $2 \mathrm{C}$ ), and the intensities of the immunofluorescence obtained with the two antibodies was compared. The average ratio of anti-PKCa-A (active) to anti-PKCa-B (total) immunofluorescence in wild type RBCs was $1.12 \pm 0.12$ ( $n=4$ mice, each with 4 technical replicates) compared to $0.70 \pm 0.15$ ( $n=3$ mice, each with 4 technical replicates) in TRPM1-KO RBCs $(P<0.0001)$, indicating that PKCa is less active in RBCs in the absence of TRPM1.

PKCa is a DAG-sensitive PKC isoform, and therefore PKCa phosphorylation can be potentiated by the DAG analogue phorbol 12-myristate 13-acetate (PMA). To confirm that PKCa is phosphorylating proteins in the retina ex vivo, we incubated freshly dissected wild type and PKCa-KO retinas in PMA before analyzing changes in protein phosphorylation by immunoblotting for PKC motif phosphoserines (PKC motif $p$-serine). PMA activation of other DAG-sensitive PKC 
isoforms expressed in the retina, such as $\mathrm{PKC} \beta$, should be relatively equivalent between WT and PKCa-KO samples and result in no difference in phosphorylation. PMA treatment resulted in a significant increase in intensity of several phosphoserine immunoreactive bands in the wild type samples at the 15, 30, and 60 min time-points compared to the PKCa-KO samples (Figure 3A) demonstrating that PMA treatment increases differential phosphorylation between WT and PKCaKO retinas. To identify sites of PMA-activated PKC phosphorylation in the wild type retina, PKC motif phosphoserine immunofluorescent labeling was performed on retina sections made from PMA-treated, light-adapted WT retinas. PMA incubation resulted in an increase in PKC motif phosphoserine immunofluorescence in presumptive RBC dendritic tips (Figure 3B).

\section{Differential protein abundance in wild type and $P K C \alpha-K O$ retina}

To identify retinal proteins whose expression is dependent on PKCa, four wild type and five PKCa-KO retinas were incubated with PMA for an hour immediately after dissection and then processed for multiplexed TMT mass spectroscopy (Figure 4). Retinas were lysed and proteins digested with trypsin before TMT labeling and LCMS/MS. Peptide identification was performed with Proteome Discoverer using SEQUEST and Percolator. From 38,384 confidently identified PSMs, there were 34,969 unique peptides corresponding to 4,435 proteins (excluding contaminants; Figure 5, S1 - Total Protein and Phosphopeptide Abundance Analysis). Differential expression (DE) statistical testing was done with edgeR using the trimmed mean of M-values normalization and an exact pairwise test. After Benjamini-Hochberg p-value corrections for multiple comparisons to establish DE false discovery rates (FDRs), we grouped proteins with significant differential expression into significance groups based on FDR thresholds: not significant (FDR $>0.10$ [4412 proteins]) low significance (0.10 $>$ FDR $>0.05$ [2 proteins]), medium significance (0.05 > FDR > 0.01 [5 proteins]), and high significance (FDR < 0.01 [16 proteins]).

Plotting the mean reporter ion intensities between wild type and PKCa-KO samples (Figure 5A) revealed a small number of significantly different protein abundances in both WT (15 
proteins) and $\mathrm{KO}$ (8 proteins). The proteins with the largest changes in abundance were KPCA (PKCa; 14-fold decrease in KO), and MTUS1 (Microtubule Associated Scaffold Protein 1; 24-fold increase in KO). A volcano plot comparing $\log _{2}$ fold change (WT / KO) with $-\log _{10}$ FDR (Figure 5B) depicts the 23 proteins passing the FDR $<0.1$ cutoff for low significance. We did not attempt any isotopic corrections for reporter ions, so the degree of downregulation for PKCa is consistent with an absence of the kinase in the knockout. A list of all significant differentially expressed proteins can be seen in Figure 5C. The sample-to-sample reproducibility of the protein abundance experiment was excellent: the WT samples had a median coefficient of variance (CV) of $11.4 \%$, the KO samples had a median CV of $15.5 \%$, and the independent CV was $13.5 \%$. Ninety-five percent of the expression changes were less than 1.25-fold different (S2 - Total Protein and Phosphopeptide Statistical Testing).

\section{Differential protein phosphorylation between wild type and PKCa-KO retina}

To examine differences in protein phosphorylation between wild type and PKCa-KO retinas, phosphopeptides were enriched from the same retinal extracts used above (described in the Methods), and phosphopeptide abundance was analyzed by multiplexed TMT mass spectroscopy. Peptide identification was performed with Proteome Discoverer using SEQUEST and Percolator, and phosphorylation localization was performed using phosphoRS. Results files were exported for post processing (filtering and aggregation) using an in-house Python script (PD1.4 TMT phospho processer.py available at https://github.com/pwilmart/PAW pipeline). The aggregated phosphopeptide reporter ion data was tested for differential expression using edgeR with multiple testing corrections, and phosphopeptides were grouped into significance groups based on differential expression FDR as described for total proteins.

We identified 1137 distinct phosphopeptides in wild type or PKCa-KO retina lysates (Figure 6, S1 - Total Protein and Phosphopeptide Abundance Analysis) 1113 were not significant $(F D R>0.10)$, seven showed low significance $(0.10>F D R>0.05)$, four medium significance $(0.05$ 
$>$ FDR $>0.01$ ), and thirteen were highly significant (FDR $<0.01)$. Of the 24 significant phosphoproteins, only two showed differential expression in the total protein abundance analysis: Dnmt3a (DNA (Cytosine-5)-Methyltransferase 3A), which was significantly more abundant in the WT samples (1.5fold), and Scg5 (Neuroendrocrine Protein 7B2), which was significantly more abundant in the KO samples (2.04-fold). Plotting WT and KO peak ion intensities (Figure 6A) highlights five phosphopeptides with much greater expression in WT than KO. A volcano plot comparing $\log _{2} \mathrm{FC}\left(\mathrm{WT} / \mathrm{KO}\right.$ ) with - $\log _{10}$ FDR (Figure 6B) shows the 24 phosphopeptides passing the FDR $<0.1$ threshold (14 increased in WT and 10 increased in KO). A full list of all significant differentially expressed phosphopeptides, along with their total protein abundance changes, can be seen in Figure 6C. The reproducibility of the peptide-centric experiment was strong: the WT samples had a median CV of $13.9 \%$, the $\mathrm{KO}$ samples had a median $\mathrm{CV}$ of $18.7 \%$, and the median $\mathrm{CV}$ independent of condition was $16.2 \%$. Ninety-five percent of the expression changes were less than 1.4-fold different (S2 - Total Protein and Phosphopeptide Statistical Testing). Annotated fragment ion spectra for the phosphorylated peptides are shown in S3 - Annotated Phosphopeptide Spectra.

Five phosphopeptides belonging to three proteins showed the largest and most significant differential abundance between the wild type and PKCa-KO samples (Figure 7). Two similar phosphopeptides were identified corresponding to BORG4 (Binder of Rho-GTPase, also called CDC42EP4) with phosphoserine residues observed at the peptide sites S6 (71-fold increase) and S8 (25-fold increase). Both peptides were generated by slightly different cleavage patterns of the same amino acid sequence with the same phosphorylated serine residue corresponding to site S64 in full-length BORG4 (Figure 7A). One phosphopeptide fragment was identified from NHERF1 $\left(\mathrm{Na}^{+} / \mathrm{H}^{+}\right.$Exchanger Regulatory Factor 1 , also called SLC9A3R1 and EBP50) with a phosphorylated serine residue at the peptide S10 position (28-fold increase) corresponding to S275 in the full-length protein (Figures 7B). The last two major significant phosphopeptides correspond to the same 10 amino acid sequence of TPBG (Trophoblast Glycoprotein, also called 
the 5T4 antigen and WAIF1) with two identified species: a doubly-phosphorylated peptide with phosphoserines at peptide S6 and S8 (13-fold increase), and a singly-phosphorylated peptide with just phosphorylated peptide S8 (2.8-fold increase). These two serine residues correspond to S422 and S424 in the C-terminal intracellular tail of TPBG (Figure 7C).

\section{Grouping of significant protein and phosphoprotein results by biological function}

Proteins identified in the total protein abundance (Figure 8A) and phosphoprotein abundance (Figure 8B) experiments were grouped into broad categories based on general biological function annotations added from Uniprot (https://github.com/pwilmart/annotations.git). PKCa-KO resulted in differential expression of proteins and phosphoproteins involved in many aspects of cellular physiology, particularly cytoskeletal rearrangement and vesicle trafficking (15 proteins), transcriptional regulation (8 proteins), and homeostasis and metabolism (5 proteins).

\section{Localization of the major PKCa-dependent phosphoproteins in the mouse retina}

We used immunoblotting and immunofluorescence confocal microscopy to examine the presence of the three most prominent PKCa-dependent phosphoprotein hits in the wild type retina: BORG4, NHERF1, and TPBG. Immunoblotting for BORG4 shows a distinct band at 38 $\mathrm{kDa}$ (Figure 9A) in agreement with the predicted molecular weight of BORG4. Immunofluorescence double-labeling of retina sections for BORG4 and PKCa shows punctate BORG4 labeling in the outer plexiform layer (OPL), but the BORG4 puncta are not strongly colocalized with PKCa (Figure 9B). This is consistent with labeling of either RBC or horizontal cell dendritic tips, as they are closely apposed to one another within the rod spherule invagination $[32,33]$. BORG4 immunofluorescence was also detected in nuclei in the inner nuclear layer (INL) and ganglion cell layer. 
Immunoblotting of retinal proteins for NHERF1 detected a strong band at $50 \mathrm{kDa}$, the predicted molecular weight of NHERF1 (Figure 9C). Immunofluorescent labeling of retina sections revealed NHERF1 immunoreactivity at the level of the photoreceptor inner segments and in the retinal pigment epithelium (RPE; Figure 9D). It was not possible to determine whether NERF1 at the level of the inner segments is due to its presence in the photoreceptors themselves or the apical microvilli of the RPE, which surround the photoreceptor outer and inner segments. No NHERF1 immunofluorescence was seen in the synaptic layers of the retina.

Immunoblotting of retina lysate for TPBG labels a broad band centered around $72 \mathrm{kDa}$ (Figure 9E). This is higher than the predicted molecular weight of $42 \mathrm{kDa}$, but is consistent with extensive glycosylation of TPBG [34]. Immunofluorescent localization of TPBG revealed strong immunoreactivity in the OPL as well as in large synaptic terminals at sublamina 5 of the inner plexiform layer (IPL; Figure 9F). In the OPL, TPBG labeling co-localizes with PKCa in RBC dendrites and cell bodies. In the IPL, TPBG immunoreactivity overlaps with all PKCa-positive RBC synaptic terminals. The immunofluorescence localization of TPBG to RBCs corroborates previous findings that TPBG mRNA is expressed primarily in RBCs [35]. The TPBG antibody also labeled a population of amacrine cells with cell bodies in the inner INL and dense dendritic projections to the middle of the IPL (Figure 9F arrows). The existence of a TPBG-positive amacrine cell population in the retina was observed by Imamura et al. in 2006 [36], but its identity has not been established.

\section{Discussion}

RBCs are able to modulate their responses to changing light conditions and evidence suggests this process is regulated in part by PKCa $[9,10,37]$. The transient physiological effect of PKCa on the RBC dendrites is presumably mediated by its kinase activity, whereby PKCadependent phosphorylation changes the activity of downstream proteins. We show that RBC 
dendrites are the main sites of light- and PKCa-dependent phosphorylation (Figure 1). Using a conformation-specific PKCa antibody and an antibody mix that recognizes phosphorylated PKC substrate motifs, our results suggest that PKCa is active in light-adapted RBC dendrites. In the dark-adapted retina, PKCa is inactive and PKC substrate phosphorylation in the OPL is significantly reduced (Figures $2 \mathrm{~A}$ and $\mathrm{B}$ ). Comparison of light-adapted wild type and PKCa-KO retinas confirmed that phosphorylation in $\mathrm{RBC}$ dendrites requires $\mathrm{PKC}$, and also indicated that phosphorylation in presumed cone bipolar cell dendrites is mediated by a different kinase. Deletion of TRPM1, a calcium-permeable cation channel responsible for signal transduction in ON-bipolar cell dendrites, also results in a significant reduction in RBC labeling with the conformation-specific PKCa antibody, suggesting that TRPM1-mediated calcium influx during the $\mathrm{RBC}$ light response is a primary source of calcium required for PKCa activation in RBC dendrites.

Differential abundance of retinal phosphopeptides between WT and PKCa-KO retinas could potentially be due to altered expression of proteins caused by the deletion of PKCa, and not due to changes in PKCa kinase activity. To identify proteins whose phosphorylation status is dependent on PKCa, but whose expression levels are unaffected, we compared both phosphoprotein and total protein abundance between WT and PKCa-KO mouse retinas using isobaric tagging and mass spectrometry. We identified over 4000 total proteins in either WT or PKCa-KO samples with 23 showing significantly different expression between groups. These proteins can be clustered into several general categories corresponding to biological ontology, most notably proteins involved in regulating cell shape and vesicle transport, transcriptional regulation, and homeostasis and metabolism. PKCa has been implicated in a variety of diverse cell signaling processes, including cell proliferation and morphology, inflammation, and tumorgenesis. Furthermore, PKCa activity has a major impact on gene expression by modulating transcription factors such as $\mathrm{CREB}, \mathrm{NF}-\kappa \mathrm{B}$, and c-REL [38]. Identifying proteins that show altered 
expression levels in PKCa-KO mouse retina could be valuable to the examination of many PKCadependent functions in RBCs, such as regulation of RBC morphology and development.

To identify RBC proteins that display PKCa-dependent phosphorylation, total retinal phosphopeptides were compared between wild type and PKCa-KO mice using isobaric tagging. Normalizations and differential expression statistical testing for isobaric tagged phosphopeotides differs from traditional phosphoproteomic experiments where replicate numbers are smaller. When replicate numbers are greater than one or two, ratios are no longer an appropriate framework for the analysis. We extended our approach of working directly with aggregated reporter ion intensities for differential protein expression to phosphopeptide datasets. Our approach enables the use of robust statistical testing from genomics packages such as edgeR [23] or Limma [39]. The background of unchanged phosphorylation levels in the enrichment experiment, like that of the total protein experiment, was sufficient in these samples to use standard normalization approaches. Our normalizations and statistical testing for both experiments demonstrates that the same analysis steps used for total protein abundance can be successfully applied to the phosphopeptide datasets (S2 - Total Protein and Phosphopeptide Statistical Testing).

Of over 1100 distinct phosphopeptides identified by multiplex TMT mass spectroscopy, 14 displayed significantly greater phosphorylation in wild type compared to PKCa-KO samples (Figure 6), suggesting their phosphorylation state is dependent on PKCa. These putative PKCadependent phosphoproteins may be phosphorylated directly by PKCa or may be phosphorylated by a different kinase whose activity is dependent on PKCa. Only one phosphoprotein, Dnmt3a, showed a significant decrease in abundance in $\mathrm{PKCa-KO}$ compared to WT in both the total protein and phosphopeptide experiments (Figures 5 and 6), indicating that reduced abundance of the Dnmt3a phosphopeptide may be due to downregulation of the protein in the KO. Three proteins, BORG4, NHERF1, and TPBG, displayed a particularly striking increase in phosphorylation in wild 
type compared to PKCa-KO samples (Figures 6 and 7). Of these proteins, BORG4 and NHERF1 are known substrates of PKCa [40,41].

Surprisingly, several of the major PKCa-dependent phosphoproteins identified in this study, including TPBG, do not conform to a strong consensus PKCa substrate motif, in which a phosphorylated serine is flanked by positively charged arginine and lysine residues (Kinexus Database; Vancouver, Canada; http://www.kinexusnet.ca). However, kinase substrate motifs are not always linear, contiguous sequences, but can also be formed structurally by bending of flexible loops that brings positively charged amino acids into proximity to the phosphorylated serine or threonine residues [42]. In the case of TPBG, the cytoplasmic domain is predicted to be unstructured and flexible, possibly allowing the phosphorylated serines at the C-terminal tail (S422 and S424) to be brought close to upstream pairs of lysines and arginines (R384 K385 and K388 and K389) to form a structural PKCa substrate motif. Alternatively, TPBG and other PKCadependent non-consensus phosphopeptides may be phosphorylated by a downstream kinase that is dependent on PKCa activity. For example, casein kinase 2 (CK2) is a serine/threonine kinase that is activated by PKCa [43,44], and whose consensus substrate motif is a serine flanked by acidic residues (Kinexus Database). Two of the phosphopeptides that showed increased abundance in wild type samples compared to PKCa-KO contain a CK2 substrate motif (TPBG and Mfap2). The same site in Mfap2 has previously been demonstrated to be phosphorylated in vitro. The C-terminals of the NR2B subunit of the NMDA receptor is similar in sequence to the Cterminus of TPBG (LSSIESDV compared to LSSNSDV), and CK2 phosphorylation of the Cterminal serine in NR2B has been demonstrated to regulate trafficking of the receptor [45]. Ten phosphopeptides were more abundant in PKCa-KO samples than in wild type. These are likely to be phosphorylated by kinases that are inhibited by PKCa, such as GSK3 $[46,47]$. The consensus substrate motif for GSK3 kinase is a serine residue with a neighboring proline (Kinexus 
Database), and several of the phosphopeptides that are increased in the PKCa-KO samples fit this motif (examples: Dyn3, Crocc, Map2, and Rtn4).

We used immunofluorescence to localize three phosphoproteins that displayed the greatest differential phosphorylation between wild type and PKCa-KO samples. Immunofluorescence labeling of BORG4 resulted in bright puncta in the OPL (Figure 9) consistent with BORG4 localization to either RBC or horizontal cell dendrites, as well as immunofluorescence over most nuclei. BORG4 belongs to a protein family (BORG1-5) that bind to the Rho GTPase CDC42 as well as to septins, a family of GTP-binding cytoskeletal proteins that are involved in regulation of cell morphology through modulation of cytoskeletal rearrangement. BORG4 contains an N-terminal CCD42/Rac Interactive Binding Motif (CRIB) and three BORG Homology (BHs) domains that are conserved across all BORG family proteins [48]. Our proteomics data indicates that the serine residue S64 in BORG4, which is located between $\mathrm{BH} 1$ and $\mathrm{BH} 2$, is phosphorylated in a PKCa-dependent manner. Phosphorylation of BORG4 S64 has been previously recognized in large-scale analyses experiments of tissue-specific phosphorylation patterns $[49,50]$ though not in retina. BORG4 and Septin-4 mRNAs have been previously found to be expressed in horizontal cells [51]. Our immunofluorescence labeling of BORG4 in retina resulted in bright puncta in the OPL consistent with BORG4 localization to either RBC or horizontal cell dendrites.

We detected NHERF1 immunofluorescence at the level of the photoreceptor inner segments in the region of the connecting cilia and in the retina pigment epithelium (RPE, Figure 9). NHERF1 is a scaffolding protein containing tandem PDZ domains and an Ezrin/Radixin/Moesin Binding (EB) domain. Our detection of strong NHERF1 immunofluorescence in the RPE consistent with previous reports localizing NHERF1 to the RPE apical microvilli [52]. NHERF1 interacts with ezrin to maintain the structure of apical microvilli on epithelia. In the RPE, NHERF1 has been implicated in retinoid recycling [52] through its 
interactions with CRALBP $[53,54]$. PKCa is also expressed in the RPE [55] where it is involved in proliferation and migration [56], phagocytosis [57], and melanin production [58]; however, the specific role of PKCa-mediated phosphorylation of NHERF1 in the RPE is unknown.

Immunofluorescence confocal microscopy localized TPBG immunoreactivity to RBC dendrites and synaptic terminals, as well as to a class of amacrine cells (Figure 9). TPBG is a heavily glycosylated type-1 transmembrane protein with a large extracellular N-terminal domain and a short C-terminal intracellular tail. The $\mathrm{N}$-terminal domain contains eight leucine-rich repeats (LRRs) and seven N-linked glycosylation sites. The C-terminal tail ends with the class-1 PDZinteracting motif (S/T X $\Phi)$ SDV [59], which our proteomics data suggests is phosphorylated in a PKCa-dependent manner. Since phosphorylation of a PDZ-interacting motif typically prevents binding of a PDZ protein [60], PKCa might be regulating interactions between TPBG and PDZ proteins by stimulating phosphorylation of its C-terminal tail. As an oncofetal antigen, TPBG is present primarily during embryonic development $[61,62]$, but is also expressed in many carcinomas (Southall et al., 1990). In the adult, TPBG is expressed in the brain, retina, and ovaries $[65,66]$. In the embryo and in cancer tissue, TPBG is involved in regulating actin polymerization [67] filopodia formation [68], and chemotaxis [69,70], and its expression in tumors is linked to increased metastatic malignancy and poor survival outcomes in cancer patients $[71,72]$. In the adult olfactory bulb, TPBG is required to stimulate the development of input-dependent dendritic arborization and synaptogenesis of newborn granule cells [36,73-75]. The role of TPBG in the retina is not yet understood; however, a recent transcriptomic classification of retinal cell types identified TPBG mRNA as being highly enriched in RBCs [35]. This is consistent with our immunofluorescent analysis which localized TPBG to RBC dendrites and synaptic terminals. 


\section{Conclusions}

The molecular mechanisms of PKCa-mediated modulation of the RBC light response have not been thoroughly explored. In this study, we have shown that PKCa phosphorylation in the retina occurs predominately in RBC dendrites in the light. Using a phosphoproteomics approach, we have identified a small number of phosphoproteins with significantly increased PKCadependent phosphorylation in the PMA-treated retina. These differentially phosphorylated proteins fall into several broad functional groups, including cytoskeleton/trafficking (4 proteins), structure and adhesion ( 2 proteins), cell signaling ( 2 proteins), transcriptional regulation ( 3 proteins), and homeostasis/metabolism (1 protein). Two strongly differentially expressed phosphoproteins, BORG4 and TPBG, are localized to the synaptic layers of the retina, and may play a role in PKCa-dependent modulation of RBC function.

\section{References}

[1] A. Berntson, R.G. Smith, W.R. Taylor, Transmission of single photon signals through a binary synapse in the mammalian retina, Vis. Neurosci. 21 (2004) 693-702.

[2] A.P. Sampath, F. Rieke, Selective transmission of single photon responses by saturation at the rod-to-rod bipolar synapse., Neuron. 41 (2004) 431-443.

[3] M.M. Abd-El-Barr, M.E. Pennesi, S.M. Saszik, A.J. Barrow, J. Lem, D.E. Bramblett, D.L. Paul, L.J. Frishman, S.M. Wu, Genetic Dissection of Rod and Cone Pathways in the DarkAdapted Mouse Retina, J. Neurophysiol. 102 (2009) 1945-1955.

[4] J.-B. Ke, Y. V Wang, B.G. Borghuis, M.S. Cembrowski, H. Riecke, W.L. Kath, J.B. Demb, J.H. Singer, Adaptation to Background Light Enables Contrast Coding at Rod Bipolar Cell Synapses, Neuron. 81 (2014) 388-401.

[5] T. Szikra, S. Trenholm, A. Drinnenberg, J. Jüttner, Z. Raics, K. Farrow, M. Biel, G. Awatramani, D.A. Clark, J.-A. Sahel, R.A. da Silveira, B. Roska, Rods in daylight act as 
relay cells for cone-driven horizontal cell-mediated surround inhibition, Nat. Publ. Gr. 17 (2014) 1728-1735.

[6] U. Greferath, U. Grünert, H. Wässle, Rod bipolar cells in the mammalian retina show protein kinase C-like immunoreactivity., J. Comp. Neurol. 301 (1990) 433-442.

[7] S. Haverkamp, F. Haeseleer, A. Hendrickson, A comparison of immunocytochemical markers to identify bipolar cell types in human and monkey retina, Vis. Neurosci. 20 (2003) 589-600.

[8] S. Haverkamp, K.K. Ghosh, A.A. Hirano, H. Wässle, Immunocytochemical description of five bipolar cell types of the mouse retina, J. Comp. Neurol. 455 (2002) 463-476.

[9] K. Ruether, A. Feigenspan, J. Pirngruber, M. Leitges, W. Baehr, O. Strauss, PKC $\{$ alpha\} is essential for the proper activation and termination of rod bipolar cell response., Investig. Ophthalmol. \&amp; Vis. Sci. 51 (2010) 6051-6058.

[10] W.-H. Xiong, J.-J. Pang, M.E. Pennesi, R.M. Duvoisin, S.M. Wu, C.W. Morgans, The Effect of PKCa on the Light Response of Rod Bipolar Cells in the Mouse Retina., Investig. Ophthalmol. Vis. Sci. 56 (2015) 4961-4974.

[11] A. Thompson, J. Schäfer, K. Kuhn, S. Kienle, J. Schwarz, G. Schmidt, T. Neumann, C. Hamon, Tandem mass tags: A novel quantification strategy for comparative analysis of complex protein mixtures by MS/MS, Anal. Chem. 75 (2003) 1895-1904.

[12] B.K. Erickson, M.P. Jedrychowski, G.C. McAlister, R.A. Everley, R. Kunz, S.P. Gygi, Evaluating multiplexed quantitative phosphopeptide analysis on a hybrid quadrupole mass filter/linear ion trap/orbitrap mass spectrometer, Anal. Chem. 87 (2015) 1241-1249.

[13] D.L. Plubell, P.A. Wilmarth, Y. Zhao, A.M. Fenton, J. Minnier, A.P. Reddy, J. Klimek, X. Yang, L.L. David, N. Pamir, Extended Multiplexing of Tandem Mass Tags (TMT) Labeling Reveals Age and High Fat Diet Specific Proteome Changes in Mouse Epididymal Adipose Tissue, Mol. Cell. Proteomics. 16 (2017) 873-890.

[14] C.W. Morgans, J. Zhang, B.G. Jeffrey, S.M. Nelson, N.S. Burke, R.M. Duvoisin, R.L. 
Brown, TRPM1 is required for the depolarizing light response in retinal ON-bipolar cells., Proc. Natl. Acad. Sci. U. S. A. 106 (2009) 19174-19178.

[15] C.T. Rueden, J. Schindelin, M.C. Hiner, B.E. DeZonia, A.E. Walter, E.T. Arena, K.W. Eliceiri, ImageJ2: ImageJ for the next generation of scientific image data, BMC Bioinformatics. 18 (2017).

[16] J. Schindelin, C.T. Rueden, M.C. Hiner, K.W. Eliceiri, The ImageJ ecosystem: An open platform for biomedical image analysis, Mol. Reprod. Dev. 82 (2015) 518-529.

[17] J.A. Paulo, F.E. Mcallister, R.A. Everley, S.A. Beausoleil, A.S. Banks, S.P. Gygi, Effects of MEK inhibitors GSK1120212 and PD0325901 in vivo using 10-plex quantitative proteomics and phosphoproteomics, Proteomics. 15 (2015) 462-473.

[18] A.N. Kettenbach, S.A. Gerber, Rapid and reproducible single-stage phosphopeptide enrichment of complex peptide mixtures: Application to general and phosphotyrosinespecific phosphoproteomics experiments, Anal. Chem. 83 (2011) 7635-7644.

[19] J.R. Eng, J.K., McCormack, A.L. \& Yates, An approach to correlate tandem mass- spectral data of peptides with amino-acid-sequences in a protein database., J. Am. Soc. Mass Spectrom. 5 (1994) 976-989.

[20] L. Käll, J.D. Canterbury, J. Weston, W.S. Noble, M.J. MacCoss, Semi-supervised learning for peptide identification from shotgun proteomics datasets, Nat. Methods. 4 (2007) 923925.

[21] T. Taus, T. Köcher, P. Pichler, C. Paschke, A. Schmidt, C. Henrich, K. Mechtler, Universal and confident phosphorylation site localization using phosphoRS, J. Proteome Res. 10 (2011) 5354-5362.

[22] N. Agrawal, R. Akbani, B.A. Aksoy, A. Ally, M. Xing, Bioconductor: open software development for computational biology and bioinformatics., Genome Biol. 11 (2010) 184199.

[23] M.D. Robinson, D.J. McCarthy, G.K. Smyth, edgeR: A Bioconductor package for 
differential expression analysis of digital gene expression data, Bioinformatics. 26 (2009) 139-140.

[24] R.C. Gentleman, V.J. Carey, D.M. Bates, B. Bolstad, M. Dettling, S. Dudoit, B. Ellis, L. Gautier, Y. Ge, J. Gentry, K. Hornik, T. Horthon, H. W., S. lacus, R. Irizarry, F. Leisch, C. Li, M. Maechler, G. Rossini A. J. Sawitzki, C. Smith, G. Smyth, L. Tierney, J.Y.H. Yang, J. Zhang, Bioconductor: open software development for computational biology and bioinformatics., Genome Biol. 5 (2004) R80.

[25] M.D. Robinson, A. Oshlack, A scaling normalization method for differential expression analysis of RNA-seq data, Genome Biol. 11 (2010) R25.

[26] B. Hochberg, Controlling the False Discovery Rate: a Practical and Powerful Approach to Multiple Testing, J. R. Stat. Soc. 57 (1995) 289-300.

[27] Y. Perez-Riverol, A. Csordas, J. Bai, M. Bernal-Llinares, S. Hewapathirana, D.J. Kundu, A. Inuganti, J. Griss, G. Mayer, M. Eisenacher, E. Pérez, J. Uszkoreit, J. Pfeuffer, T. Sachsenberg, Ş. Yılmaz, S. Tiwary, J. Cox, E. Audain, M. Walzer, A.F. Jarnuczak, T. Ternent, A. Brazma, J.A. Vizcaíno, The PRIDE database and related tools and resources in 2019: improving support for quantification data, Nucleic Acids Res. 47 (2019) D422D450.

[28] R. Gabriel, J. Lesauter, R. Silver, A. Garcia-Espaa, P. Witkovsky, Diurnal and circadian variation of protein kinase C immunoreactivity in the rat retina, J. Comp. Neurol. 439 (2001) $140-150$.

[29] Y. Nishizuka, Intracellular signaling by hydrolysis of phospholipids and activation of protein kinase C, Science (80-. ). 258 (1992) 607-614.

[30] Y. Shen, M.A.F. Rampino, R.C. Carroll, S. Nawy, G-protein-mediated inhibition of the Trp channel TRPM1 requires the G dimer, Proc. Natl. Acad. Sci. 109 (2012) 8752-8757.

[31] C. Koike, T. Numata, H. Ueda, Y. Mori, T. Furukawa, TRPM1: a vertebrate TRP channel responsible for retinal ON bipolar function., Cell Calcium. 48 (2010) 95-101. 
[32] B.B. Boycott, J.E. Dowling, H. Kolb, Organization of the Primate Retina: Light Microscopy, Philos. Trans. R. Soc. B Biol. Sci. 255 (1969) 109-184.

[33] J.E.. Dowling, B.. B.. Boycott, Organization of the Primate Retina: Electron Microscopy, Proc. R. Soc. London, Ser. B , Biol. Sci. 166 (1966) 80-111.

[34] D.M. Shaw, A.M. Woods, K.A. Myers, C. Westwater, V. Rahi-Saund, M.J. Davies, D. V Renouf, E.F. Hounsell, P.L. Stern, Glycosylation and epitope mapping of the 5T4 glycoprotein oncofoetal antigen., Biochem. J. 363 (2002) 137-145.

[35] K. Shekhar, S.W. Lapan, I.E. Whitney, N.M. Tran, E.Z. Macosko, M. Kowalczyk, X. Adiconis, J.Z. Levin, J. Nemesh, M. Goldman, S.A. McCarroll, C.L. Cepko, A. Regev, J.R. Sanes, Comprehensive Classification of Retinal Bipolar Neurons by Single-Cell Transcriptomics, Cell. 166 (2016) 1308-1323.

[36] F. Imamura, H. Nagao, H. Naritsuka, Y. Murata, H. Taniguchi, K. Mori, A leucine-rich repeat membrane protein, $5 \mathrm{~T} 4$, is expressed by a subtype of granule cells with dendritic arbors in specific strata of the mouse olfactory bulb, J. Comp. Neurol. 495 (2006) 754-768.

[37] M.A.F. Rampino, S.A. Nawy, Relief of $\mathrm{Mg}^{2+}$-dependent inhibition of TRPM1 by PKCa at the rod bipolar cell synapse., J. Neurosci. 31 (2011) 13596-13603.

[38] R. Garg, M.C. Caino, M.G. Kazanietz, Regulation of Transcriptional Networks by PKC Isozymes: Identification of c-Rel as a Key Transcription Factor for PKC-Regulated Genes, PLoS One. 8 (2013) e67319.

[39] M.E. Ritchie, B. Phipson, D. Wu, Y. Hu, C.W. Law, W. Shi, G.K. Smyth, Limma powers differential expression analyses for RNA-sequencing and microarray studies, Nucleic Acids Res. 43 (2015) e47.

[40] X. Chen, X. Zhao, T.P. Abeyweera, S.A. Rotenberg, Analysis of substrates of protein kinase $\mathrm{C}$ isoforms in human breast cells by the traceable kinase method, Biochemistry. 51 (2012) 7087-7097.

[41] D. Garbett, D.P. LaLonde, A. Bretscher, The scaffolding protein EBP50 regulates 
microvillar assembly in a phosphorylation-dependent manner, J. Cell Biol. 191 (2010) 397413.

[42] M.L. Duarte, D.A. Pena, F.A.N. Ferraz, D.A. Berti, T.J.P. Sobreira, H.M. Costa-Junior, M.M.A. Baqui, M.H. Disatnik, J. Xavier-Neto, P.S.L. De Oliveira, D. Schechtman, Protein folding creates structure-based, noncontiguous consensus phosphorylation motifs recognized by kinases, Sci. Signal. 7 (2014).

[43] N. Malkani, K. Biggar, M.A. Shehab, S.S.C. Li, T. Jansson, M.B. Gupta, Increased IGFBP1 phosphorylation in response to leucine deprivation is mediated by CK2 and PKC, Mol. Cell. Endocrinol. 425 (2016) 48-60.

[44] Y.-H. Lee, J.-W. Park, Y.-S. Bae, Regulation of protein kinase CK2 catalytic activity by protein kinase C and phospholipase D2., Biochimie. 121 (2016) 131-139.

[45] H.J. Chung, Y.H. Huang, L.-F. Lau, R.L. Huganir, Regulation of the NMDA Receptor Complex and Trafficking by Activity-Dependent Phosphorylation of the NR2B Subunit PDZ Ligand, J. Neurosci. 24 (2004) 10248-10259.

[46] S.F. Moore, M.T.J. Van Den Bosch, R.W. Hunter, K. Sakamoto, A.W. Poole, I. Hers, Dual regulation of glycogen synthase kinase $3($ GSK3) $\alpha / \beta$ by protein kinase $C(P K C) \alpha$ and Akt promotes thrombin-mediated integrin $\alpha \| b \beta 3$ activation and granule secretion in platelets, J. Biol. Chem. 288 (2013) 3918-3928.

[47] Z.-C. Liu, X.-H. Chen, H.-X. Song, H.-S. Wang, G. Zhang, H. Wang, D.-Y. Chen, R. Fang, H. Liu, S.-H. Cai, J. Du, Snail regulated by PKC/GSK-3ß pathway is crucial for EGFinduced epithelial-mesenchymal transition (EMT) of cancer cells., Cell Tissue Res. 358 (2014) 491-502.

[48] A.J. Farrugia, F. Calvo, The Borg family of Cdc42 effector proteins Cdc42EP1-5, Biochem. Soc. Trans. 44 (2016) 1709-1716.

[49] J. Villen, S.A. Beausoleil, S.A. Gerber, S.P. Gygi, Large-scale phosphorylation analysis of mouse liver, Proc. Natl. Acad. Sci. 104 (2007) 1488-1493. 
[50] E.L. Huttlin, M.P. Jedrychowski, J.E. Elias, T. Goswami, R. Rad, S.A. Beausoleil, J. Villén, W. Haas, M.E. Sowa, S.P. Gygi, A tissue-specific atlas of mouse protein phosphorylation and expression, Cell. 143 (2010) 1174-1189.

[51] S. Blackshaw, S. Harpavat, J. Trimarchi, L. Cai, H. Huang, W.P. Kuo, G. Weber, K. Lee, R.E. Fraioli, S.H. Cho, R. Yung, E. Asch, L. Ohno-Machado, W.H. Wong, C.L. Cepko, Genomic analysis of mouse retinal development, PLoS Biol. 2 (2004) E247.

[52] J. Huang, D.E. Possin, J.C. Saari, Localizations of visual cycle components in retinal pigment epithelium, Mol. Vis. 15 (2009) 223-234.

[53] V.L. Bonilha, S.K. Bhattacharya, K.A. West, J.S. Crabb, J. Sun, M.E. Rayborn, M. Nawrot, J.C. Saari, J.W. Crabb, Support for a proposed retinoid-processing protein complex in apical retinal pigment epithelium, Exp. Eye Res. 79 (2004) 419-422.

[54] M. Nawrot, K. West, J. Huang, D.E. Possin, A. Bretscher, J.W. Crabb, J.C. Saari, Cellular Retinaldehyde-Binding Protein Interacts with ERM-Binding Phosphoprotein 50 in Retinal Pigment Epithelium, Investig. Ophthalmol. Vis. Sci. 45 (2004) 393-401.

[55] K. Yu, P. Ma, J. Ge, C.D. Willey, P. Yang, Z. Wang, Q. Gao, Expression of protein kinase C isoforms in cultured human retinal pigment epithelial cells., Graefe's Arch. Clin. Exp. Ophthalmol. 254 (2007) 993-999.

[56] H. Kishi, H.K. Mishima, U. Yamashita, Growth regulation of retinal pigment epithelial (RPE) cells in vitro, Curr. Eye Res. 13 (1994) 661-668.

[57] S.J. Sheu, T. Sakamoto, R. Osusky, H.M. Wang, T.E. Ogden, S.J. Ryan, D.R. Hinton, R. Gopalakrishna, Transforming growth factor- $\beta$ regulates human retinal pigment epithelial cell phagocytosis by influencing a protein kinase C-dependent pathway, Graefe's Arch. Clin. Exp. Ophthalmol. 232 (1994) 695-701.

[58] H. Kishi, H.K. Mishima, U. Yamashita, Involvement of the protein kinase pathway in melanin synthesis by chick retinal pigment epithelial cells, Cell Biol. Int. 24 (2000) 79-83.

[59] Y. Zhao, T. Malinauskas, K. Harlos, E.Y. Jones, Structural insights into the inhibition of Wnt 
signaling by cancer antigen 5T4/Wnt-activated inhibitory factor 1., Structure. 22 (2014) 612-620.

[60] H.J. Lee, J.J. Zheng, PDZ domains and their binding partners: Structure, specificity, and modification, Cell Commun. Signal. 8 (2010).

[61] C.M. Ward, A.M. Eastham, P.L. Stern, Cell surface 5T4 antigen is transiently upregulated during early human embryonic stem cell differentiation: Effect of 5T4 phenotype on neural lineage formation, Exp. Cell Res. 312 (2006) 1713-1726.

[62] C.M. Ward, K. Barrow, A.M. Woods, P.L. Stern, The 5T4 oncofoetal antigen is an early differentiation marker of mouse ES cells and its absence is a useful means to assess pluripotency., J. Cell Sci. 116 (2003) 4533-4542.

[63] P.J. Southall, G.M. Boxer, K.D. Bagshawe, N. Hole, M. Bromley, P.L. Stern, Immunohistological distribution of 5T4 antigen in normal and malignant tissues., Br. J. Cancer. 61 (1990) 89-95.

[64] N. Hole, P.L. Stern, A 72 kD trophoblast glycoprotein defined by a monoclonal antibody., Br. J. Cancer. 57 (1988) 239-246.

[65] K.M. Barrow, C.M. Ward, J. Rutter, S. Ali, P.L. Stern, Embryonic expression of murine 5T4 oncofoetal antigen is associated with morphogenetic events at implantation and in developing epithelia., Dev. Dyn. 233 (2005) 1535-1545.

[66] K.W. King, F.C. Sheppard, C. Westwater, P.L. Stern, K.A. Myers, Organisation of the mouse and human 5T4 oncofoetal leucine-rich glycoprotein genes and expression in foetal and adult murine tissues, Biochim. Biophys. Acta - Gene Struct. Expr. 1445 (1999) 257270.

[67] A. Awan, M.R. Lucic, D.M. Shaw, F. Sheppard, C. Westwater, S.A. Lyons, P.L. Stern, 5 T4 Interacts with TIP-2/GIPC, a PDZ Protein, with Implications for Metastasis, Biochem. Biophys. Res. Commun. 290 (2002) 1030-1036.

[68] C.J. Carsberg, K.A. Myers, G.S. Evans, T.D. Allen, P.L. Stern, Metastasis-associated 5T4 
oncofoetal antigen is concentrated at microvillus projections of the plasma membrane., J. Cell Sci. 108 (1995) 2905-2916.

[69] O.J. McGinn, G. Marinov, S. Sawan, P.L. Stern, CXCL12 receptor preference, signal transduction, biological response and the expression of 5T4 oncofoetal glycoprotein, J. Cell Sci. 125 (2012) 5467-5478.

[70] T.D. Southgate, O.J. McGinn, F. V Castro, A.J. Rutkowski, M. Al-Muftah, G. Marinov, G.J. Smethurst, D. Shaw, C.M. Ward, C.J. Miller, P.L. Stern, CXCR4 Mediated Chemotaxis Is Regulated by 5 T4 Oncofetal Glycoprotein in Mouse Embryonic Cells, PLoS One. 5 (2010) e9982-20.

[71] T. Pukrop, C. Binder, The complex pathways of Wnt 5a in cancer progression, J. Mol. Med. 86 (2008) 259-266.

[72] A.T. Weeraratna, Y. Jiang, G. Hostetter, K. Rosenblatt, P. Duray, M. Bittner, J.M. Trent, Wnt5a signaling directly affects cell motility and invasion of metastatic melanoma., Cancer Cell. 1 (2002) 279-288.

[73] S. Yoshihara, H. Takahashi, N. Nishimura, H. Naritsuka, T. Shirao, H. Hirai, Y. Yoshihara, K. Mori, P.L. Stern, A. Tsuboi, 5T4 glycoprotein regulates the sensory input-dependent development of a specific subtype of newborn interneurons in the mouse olfactory bulb., J. Neurosci. 32 (2012) 2217-2226.

[74] H. Takahashi, Y. Ogawa, S. i Yoshihara, R. Asahina, M. Kinoshita, T. Kitano, M. Kitsuki, K. Tatsumi, M. Okuda, K. Tatsumi, A. Wanaka, H. Hirai, P.L. Stern, A. Tsuboi, A Subtype of Olfactory Bulb Interneurons Is Required for Odor Detection and Discrimination Behaviors, J. Neurosci. 36 (2016) 8210-8227.

[75] S. Yoshihara, H. Takahashi, A. Tsuboi, Molecular Mechanisms Regulating the Dendritic Development of Newborn Olfactory Bulb Interneurons in a Sensory Experience-Dependent Manner, Front. Neurosci. 9 (2016) 134-139. 


\section{Figure Legends}

Figure 1: Phosphoserine labeling in the OPL is reduced in PKCa-KO retina. (A) Immunofluorescence confocal images of mouse retina sections from wild type and PKCa-KO retinas labeled with an antibody against phosphoserine residues within canonical PKC motif phosphoserine (PKC motif p-serine). (B) Images of PKC motif p-serine immunofluorescence in the outer plexiform layer of wild type and PKCa knockout (KO) retinas in obliquely cut sections. In both $\mathrm{A}$ and $\mathrm{B}$, white arrows indicate labeling associated with presumptive cone pedicles. Scale bars: $20 \mu \mathrm{m}$. OPL: outer plexiform layer; IPL: inner plexiform layer.

Figure 2: PKCa is active in light-adapted RBCs. (A) Light- and dark-adapted mouse retina sections double-labeled with two antibodies against PKCa; conformation-specific PKCa-A (green) binds only active PKCa, while PKCa-B (magenta) binds to both active and inactive PKCa. (B) Sections from light-adapted and dark-adapted mouse retina labeled with an antibody mixture against PKC motif phosphoserines (PKC motif p-serine). (C) WT and TRPM1-KO mouse retina sections double-labeled with conformation-specific PKCa-A (green) and conformation nonspecific PKCa-B (magenta). Scale bars: $10 \mu \mathrm{m}$. OPL: outer plexiform layer.

Figure 3: PMA increases phosphorylation by PKC isoforms in the mouse retina. (A) Wild type (WT) and PKCa knockout (PKCa-KO) retinas were incubated in PMA for 0, 15, 30, and 60 min followed by western blotting with an antibody against PKC motif phosphoserines. Arrows indicate candidate PKCa phosphorylation targets. (B) Immunofluorescent PKC motif phosphoserine labeling of wild type mouse OPL from retinas that were incubated with and without PMA for $1 \mathrm{hr}$. Scale bar: $10 \mu \mathrm{m}$. PMA: phorbol 12-myristate 13-acetate; OPL: outer plexiform layer. 
Figure 4: Experimental workflow of total protein and phosphopeptide identification. Wild type $(n=4)$ and PKCa knockout $(K O)(n=5)$ retinas were extracted and treated with PMA before lysis and trypsin digestion. A small fraction of each sample was removed for total protein analysis, while the rest of the samples underwent phosphopeptide enrichment. Following TMT labeling, samples were combined and analyzed by LCMS/MS. Tandem mass spectrometry data was collected on an Orbitrap Fusion and proteins were identified using Proteome Discoverer (SEQUEST and Percolator). Phosphorylation site localization was scored using phosphoRS, and reporter ion intensities were filtered and aggregated with an in-house Python script. TMT reporter ion intensities from total proteins or from phosphopeptides were tested for differential expression using the Bioconductor package edgeR. The presence of representative phosphoproteins was validated in the retina by western blot and confocal immunofluorescence microscopy. PMA: phorbol 12-myristate 13-acetate.

Figure 5: Identification of differentially expressed total proteins. (A) Scatter plot of peak reporter ion intensities from wild type (WT) and PKCa knockout (KO) total protein abundance samples. The dotted line corresponds to an FC (WT / KO) of 1 , with FC $>1$ corresponding to increased abundance in WT vs $\mathrm{KO}$, and $\mathrm{FC}<1$ corresponding to increased abundance in $\mathrm{KO}$ vs WT. (B) Volcano plot of $\log _{2}$ FC and $-\log _{10}$ FDR. The dotted lines correspond to FC $=1$ and FDR $=0.1$ FDR $<0.1$ corresponds to proteins passing the low significance threshold, and FDR $>0.1$ corresponds to proteins failing the low significance threshold. The low significance (orange) threshold was 0.1 , the med significance threshold (green) was 0.05 , and the high significance threshold (blue) was 0.01. (C) Table of all differentially abundant proteins with an FDR lower than the low significance threshold of 0.1 . Fold change was calculated by dividing the mean reporter ion intensities of each protein from the genotype with higher abundance by that with lower abundance. Colors correspond to DE FDR thresholds. DE: differential expression; FC: fold change; FDR: false discovery rate. 
Figure 6: Identification of differentially expressed phosphopeptides. (A) Scatter plot of peak reporter ion intensities from wild type (WT) and PKCa knockout (KO) phosphopeptide abundance samples. The dotted line corresponds to an FC (WT / KO) of 1 , with FC $>1$ corresponding to increased abundance in WT vs $\mathrm{KO}$, and $\mathrm{FC}<1$ corresponding to increased abundance in $\mathrm{KO}$ vs WT. (B) Volcano plot of $\log _{2}$ FC and $-\log _{10}$ FDR. The dotted lines correspond to FC $=1$ and FDR $=0.1$. FDR $<0.1$ corresponds to proteins passing the low significance threshold, and FDR $>0.1$ corresponds to proteins failing the low significance threshold. The low significance (orange) threshold was 0.1 , the med significance threshold (green) was 0.05 , and the high significance threshold (blue) was 0.01. (C) Table of all differentially abundance phosphopeptides with an FDR lower than the low significance threshold of 0.1 . In the phosphopeptide sequence column, phosphorylated residues are in red. In the Total Protein Fold Change column, negative values indicate an increased abundance in the KO samples. Fold change was calculated by dividing the mean reporter ion intensities of each protein from the genotype with higher abundance by that with lower abundance. Fold change and DE FDR values were taken from the phosphopeptide abundance experiment and the total protein abundance experiment. Colors correspond to DE FDR thresholds. DE: differential expression; FC: fold change; FDR: false discovery rate.

Figure 7: TMT data from representative phosphoproteins. Reporter ion intensity values from each TMT channel for the five phosphopeptide fragments with the largest differential expression between WT ( $n=4)$ and PKCa-KO ( $n=5)$ : two from BORG4 (orange), one from NHERF1 (green), and two from TPBG (blue). For statistical significance of differential expression analysis of mean WT (black) and mean KO (red) reporter ion intensities, see S1 - Total Protein and Phosphopeptide Abundance Analysis. (A) Two phosphopeptide fragments from an overlapping region of BORG4, each containing a phosphorylated serine corresponding to $\mathrm{S} 64$ on the fulllength protein. (B) One phosphopeptide fragment from NHERF1 with a phosphorylated serine 
corresponding to S275 on the full-length protein. (C) Two phosphopeptide fragments from the Cterminal tail of TPBG with two similar phosphorylation patterns: one with two phosphoserines corresponding to S422 and S424, and one with a single phosphoserine corresponding to S424 of the full-length protein.

Figure 8: Significant total proteins and phosphoproteins grouped by biological function. Table of genes of identified total proteins $(A)$ and phosphoproteins $(B)$ with significant differential abundance between wild type and PKCa-KO samples grouped into broad categories based on general biological function gathered from Uniprot protein annotations.

Figure 9: Validation of representative phosphoproteins in the mouse retina. (A) Immunoblot of retinal lysate shows a band corresponding to BORG4 at $\sim 38 \mathrm{kDa}$. (B) Confocal microscopy analysis of BORG4 and PKCa immunoreactivity in the retina. (C) Immunoblot of retinal lysate shows a band corresponding to NHERF1 at $\sim 50 \mathrm{kDa}$. (D) Confocal microscopy analysis of NHERF1 immunoreactivity in the retina with retinal layers labeled with DAPI. (E) Immunoblot of retinal lysate shows a smear corresponding to glycosylated TPBG at $\sim 72 \mathrm{kDa}$. (F) Confocal microscopy analysis of TPBG and PKCa immunoreactivity in the retina. Scale bars: $20 \mu \mathrm{m}$. RPE: retinal pigment epithelium; OS: outer segments; IS: inner segments; ONL: outer nuclear layer; OPL: outer plexiform layer; INL: inner nuclear layer; IPL: inner plexiform layer; GCL: ganglion cell layer. 


\section{Figure 1}

\section{A \\ Wild type}

$\frac{1}{0}$

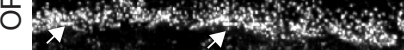

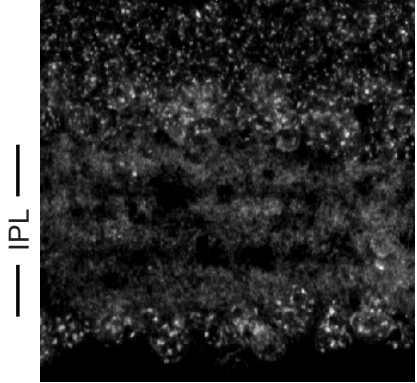

B

\section{PKCa-KO}
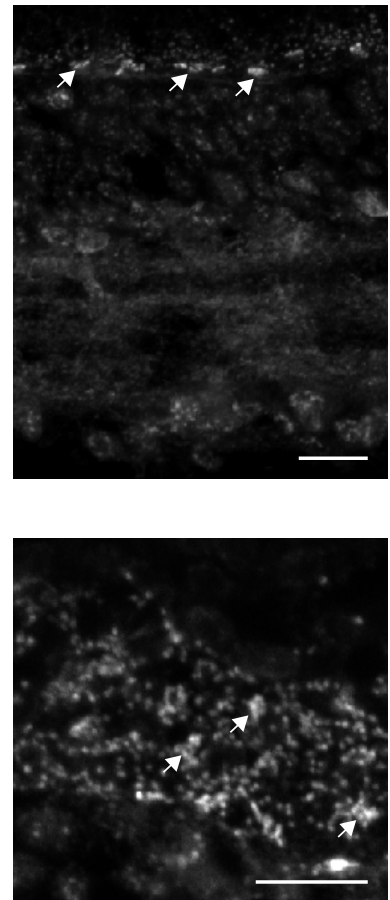
Figure 2

A Light adapted

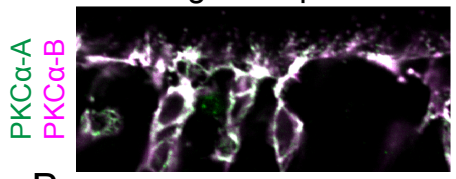

B

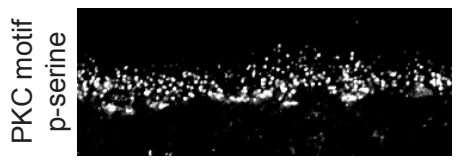

C Wild type

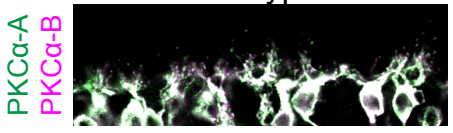

Dark adapted
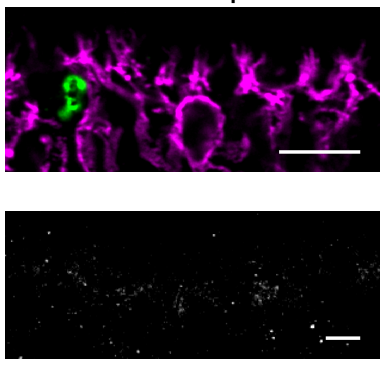

TRPM1-KO

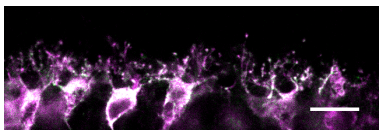




\section{Figure 3}

A

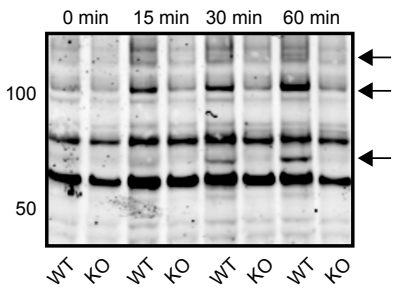

- PMA

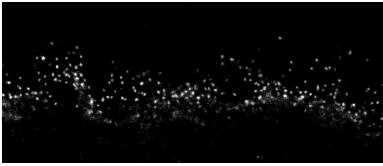

+ PMA

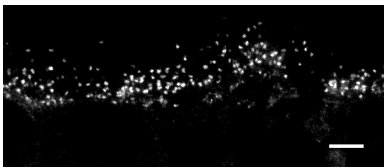




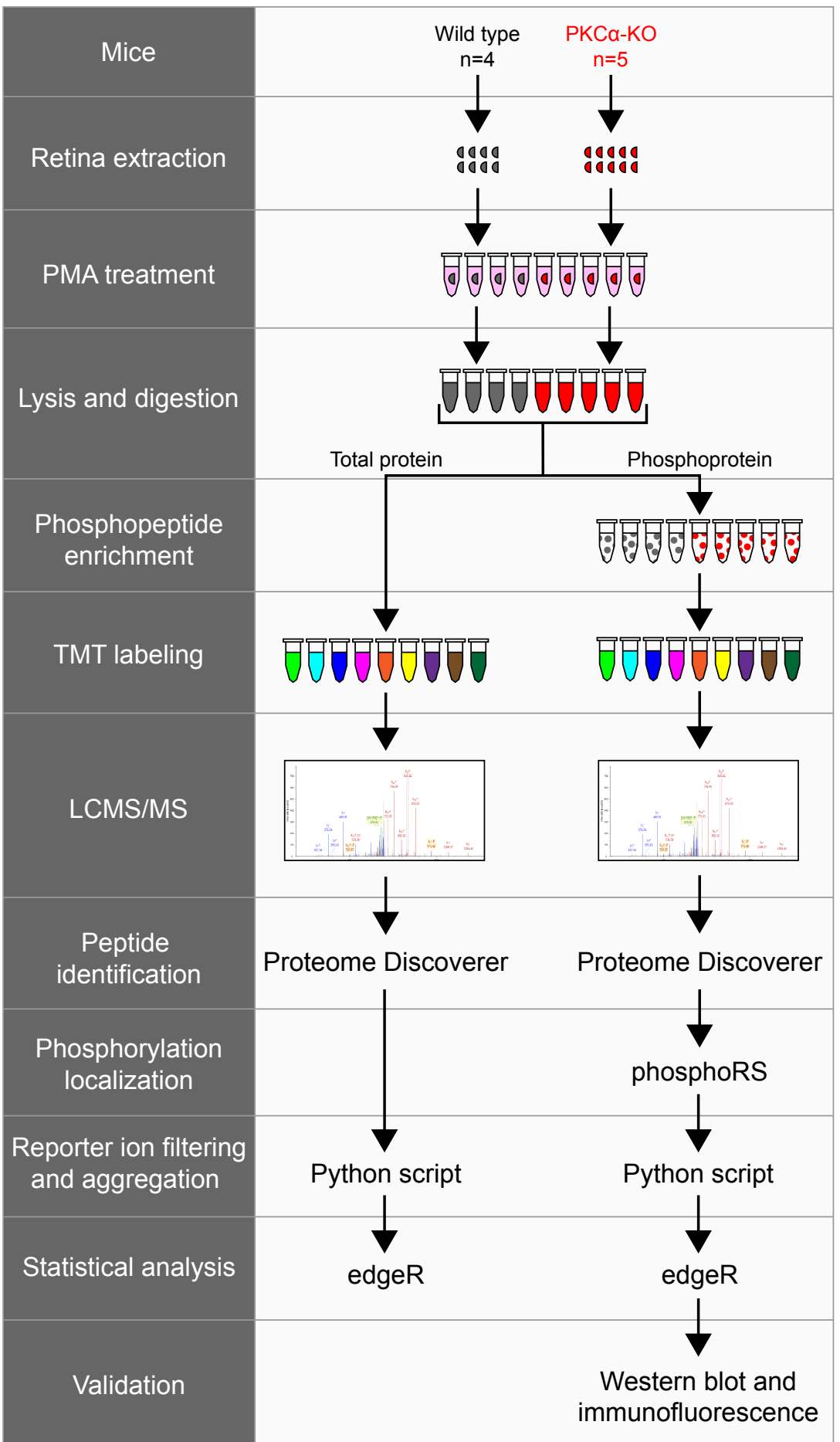


A

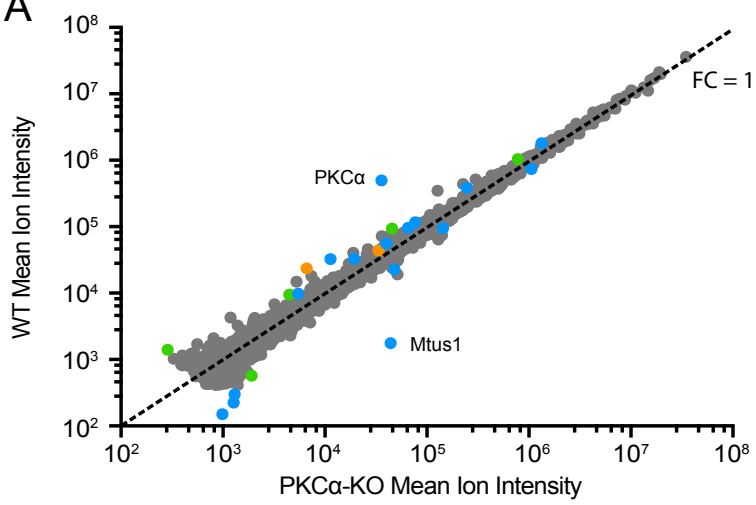

B

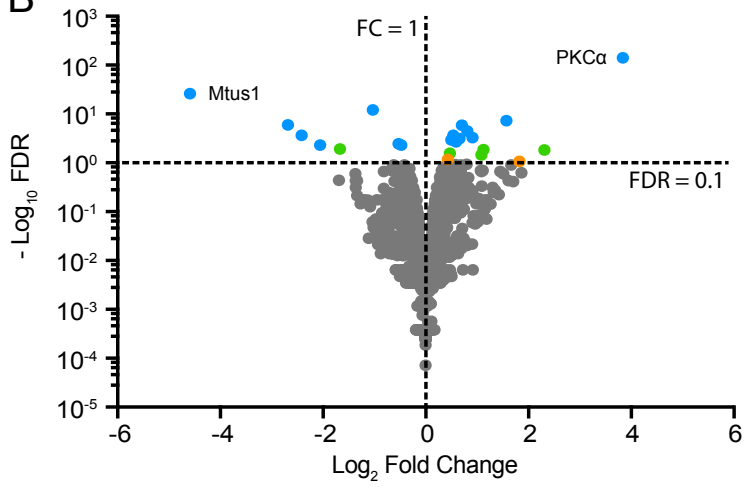

C

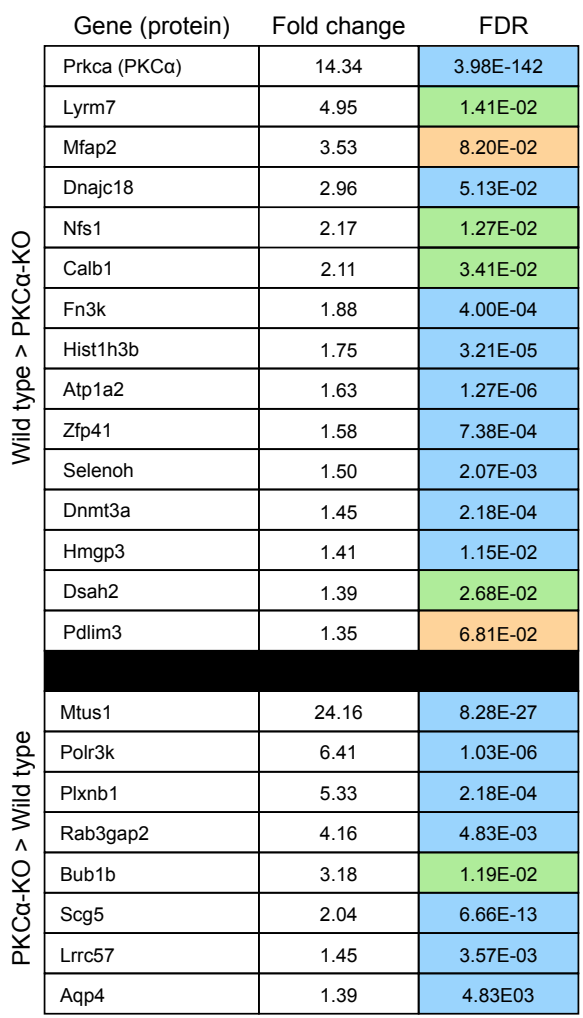

Total protein abundance Gene (protein) Fold change FDR

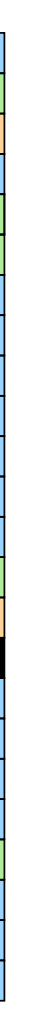




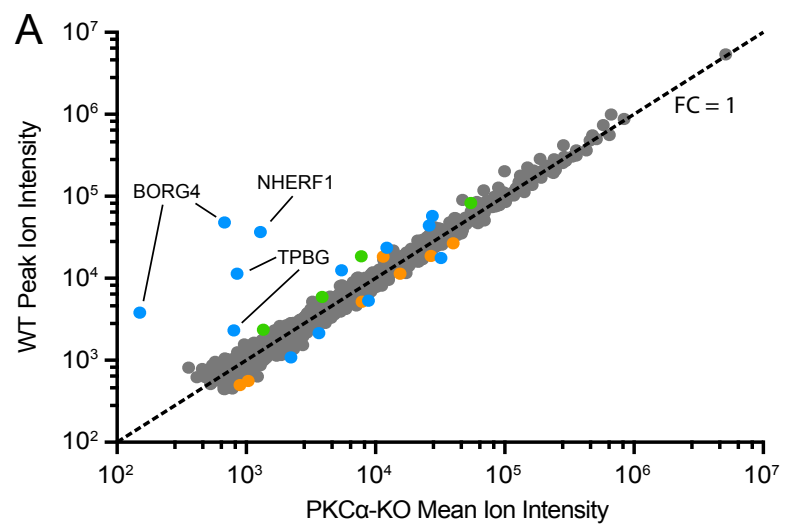

Not Significant Low Significance

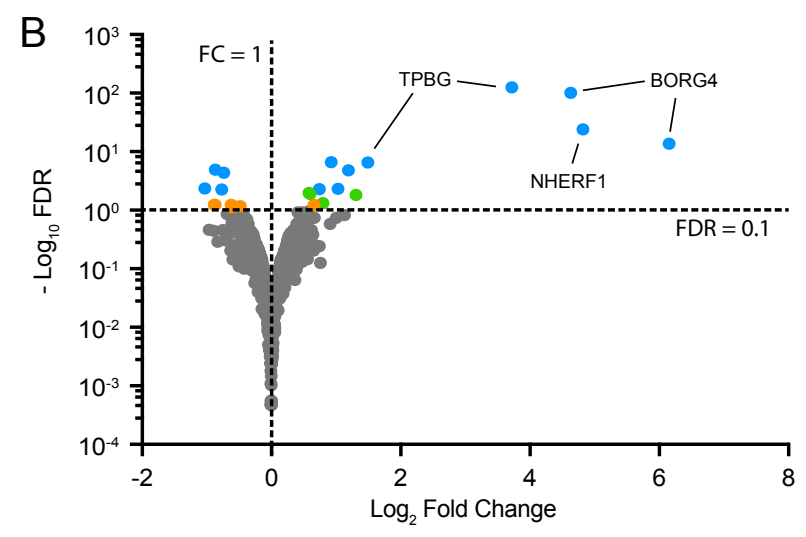

Med Significance High Significance
C

\begin{tabular}{|c|c|c|c|c|c|}
\hline \multirow[b]{2}{*}{ Phosphopeptide sequence } & \multirow[b]{2}{*}{ Gene (protein) } & \multicolumn{2}{|c|}{ Phosphopeptide } & \multicolumn{2}{|c|}{ Total protein } \\
\hline & & Fold change & FDR & Fold change & FDR \\
\hline EADDESLDEQASASKLSLLSR & Cdc42ep4 (BORG4) & 71.12 & $2.98 \mathrm{E}-14$ & 1.00 & $9.99 \mathrm{E}-01$ \\
\hline EALVEPASESPRPALAR & Slc9a3r1 (NHERF1) & 28.29 & $1.57 \mathrm{E}-24$ & 1.02 & $9.93 \mathrm{E}-01$ \\
\hline AREADDESLDEQASASKLSLLSR & Cdc42ep4 (BORG4) & 24.68 & $3.48 \mathrm{E}-103$ & 1.00 & 9.99E-01 \\
\hline LTNLSSNSDV & Tpbg & 13.20 & 1.30E-127 & 1.09 & $9.89 \mathrm{E}-01$ \\
\hline LTNLSSNSDV & Tpbg & 2.82 & 3.06E-07 & 1.09 & 9.90E-01 \\
\hline SATRVIGGPVTPR & Pde6g & 2.48 & 1.64E-02 & -1.11 & $9.69 \mathrm{E}-01$ \\
\hline SLRRQQQPCMEPPESQLEPK & Znf512 & 2.29 & 1.63E-05 & -1.03 & $9.92 \mathrm{E}-01$ \\
\hline RSSVRPGVVVPR & Ankrd33b & 2.05 & $4.84 \mathrm{E}-03$ & 1.08 & $9.52 \mathrm{E}-01$ \\
\hline RLSTHSPFR & Epb41 & 1.90 & $2.39 \mathrm{E}-07$ & 1.02 & $9.98 \mathrm{E}-01$ \\
\hline SLAALDALNTDDENDEEEYEAWKVR & Dnmt3a & 1.73 & 4.65E-02 & -1.45 & $2.18 \mathrm{E}-04$ \\
\hline RVSNRGLAGTTIR & Amer2 & 1.68 & $5.18 \mathrm{E}-03$ & -1.08 & 9.63E-01 \\
\hline DGRGAAQNIIPASTGAAK & Gapdh & 1.58 & $5.91 \mathrm{E}-02$ & 1.06 & 9.67E-01 \\
\hline SEPQPEEGSPAAQK & Epb4112 & 1.51 & 1.17E-02 & 1.04 & 9.89E-01 \\
\hline QRSYNLVVAK & Mfap1 & 1.49 & $9.81 \mathrm{E}-03$ & -1.11 & 8.82E-01 \\
\hline EGDGSATTDAAPATSPKAEEPSKAGDAPSEEK & Gap43 & 2.04 & $4.52 \mathrm{E}-03$ & 1.13 & $8.85 \mathrm{E}-01$ \\
\hline SPPPSPTTQR & Dnm3 & 1.85 & $5.80 \mathrm{E}-02$ & 1.03 & $9.92 \mathrm{E}-01$ \\
\hline ARPSQLPEQSSSAQQNGSVSDISPVQAAK & Kif2a & 1.83 & $5.78 \mathrm{E}-02$ & -1.00 & $9.98 \mathrm{E}-01$ \\
\hline SSASVSLPPGTPEK & Crocc & 1.83 & $1.16 \mathrm{E}-05$ & 1.13 & $6.64 \mathrm{E}-01$ \\
\hline ARVDHGAEIITQSPSRSSVASPR & Map2 & 1.71 & $5.39 \mathrm{E}-03$ & 1.05 & 9.89E-01 \\
\hline SVPHFSEEEKEAE & Scg5 & 1.67 & 4.37E-05 & 2.04 & $6.66 \mathrm{E}-13$ \\
\hline SFSLGDLSHSPQTAQHVER & Ccdc177 & 1.54 & $5.78 \mathrm{E}-02$ & 1.24 & 3.53E-01 \\
\hline SLTNSHLEKR & Slc39a1 & 1.53 & 7.55E-02 & NA & NA \\
\hline GPLPAAPPTAPERQPSWER & Rtn4 & 1.47 & 7.02E-02 & 1.06 & $9.78 \mathrm{E}-01$ \\
\hline SADRRLSGAQAELALQEESVR & Crocc & 1.40 & $6.77 \mathrm{E}-02$ & 1.13 & $6.64 \mathrm{E}-01$ \\
\hline
\end{tabular}

\section{Phosphoprotein abundance}


A

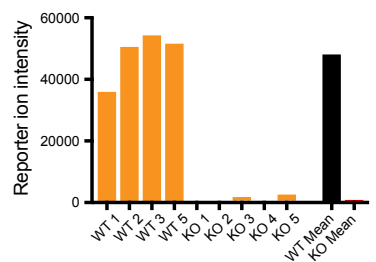

S64

NT -

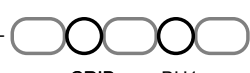

CRIB
$\mathrm{BH} 1$

\section{AREADDESLDEQASASKLSLLSR}

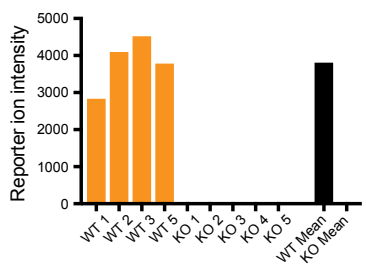

B

EALVEPASESPRPALAR

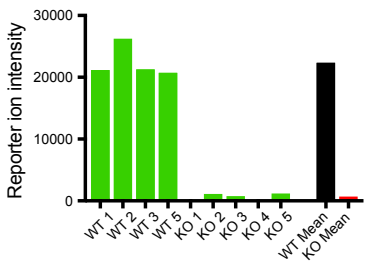

NT -

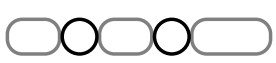

PDZ1 PDZ2

ITNLSSNSDV

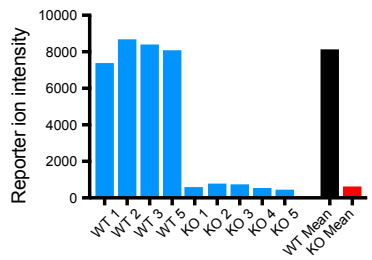

Extracellular

LRRs

NT

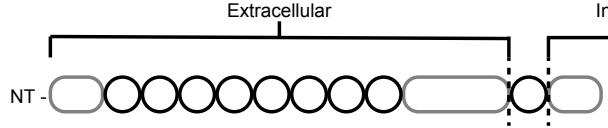

S275

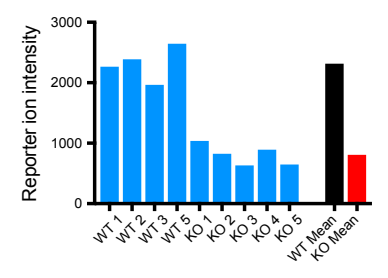

Intracellular

$\mathrm{BH} 2$

$\mathrm{BH} 3$

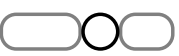

EB - CT

\section{ITNLSSNSDV}

S422 S424

ITNLSSNSDV - CT 


\section{Significant Total Proteins}

\begin{tabular}{|c|c|c|c|c|c|c|c|}
\hline & Cytoskeleton/Trafficking & ECM/Adhesion & Cell Signaling & Transcriptional Regulation & $\begin{array}{c}\text { Homeostasis/ } \\
\text { Metabolism }\end{array}$ & Chaperone & Transporter \\
\hline & $\begin{array}{l}\text { Mfap2 } \\
\text { Pdlim3 }\end{array}$ & & $\begin{array}{l}\text { Prkca } \\
\text { Calb1 }\end{array}$ & $\begin{array}{c}\text { Dnajc17 } \\
\text { Zfp41 } \\
\text { Dnmt3a } \\
\text { Hmgb3 }\end{array}$ & $\begin{array}{l}\text { Nfs1 } \\
\text { Fn3k } \\
\text { Selenoh } \\
\text { Dsah2 }\end{array}$ & $\begin{array}{c}\text { Lyrm7 } \\
\text { Hist1h3b }\end{array}$ & Atp1a2 \\
\hline & $\begin{array}{l}\text { Mtus1 } \\
\text { Plxnb1 } \\
\text { Bub1b }\end{array}$ & Lrrc57 & Rab3gap2 & Polr3k & & Scg5 & Aqp4 \\
\hline
\end{tabular}

B

\begin{tabular}{|c|c|c|c|c|c|c|}
\hline Cytoskeleton/Trafficking & ECM/Adhesion & Cell Signaling & Transcriptional Regulation & $\begin{array}{l}\text { Homeostasis/ } \\
\text { Metabolism }\end{array}$ & Chaperone & Transporter \\
\hline $\begin{array}{c}\text { Borg4 } \\
\text { Nherf1 } \\
\text { Epb41 } \\
\text { Epb41l2 }\end{array}$ & $\begin{array}{c}\text { Tpbg } \\
\text { Mfap1a }\end{array}$ & $\begin{array}{l}\text { Pde6g } \\
\text { Amer2 }\end{array}$ & $\begin{array}{c}\text { Znf512 } \\
\text { Ankrd33b } \\
\text { Dnmt3a }\end{array}$ & Gapdh & & \\
\hline $\begin{array}{c}\text { Gap43 } \\
\text { Dnm3 } \\
\text { Kif2a } \\
\text { Crocc } \\
\text { Map2 } \\
\text { Rtn4 }\end{array}$ & & & Ccdc177 & & Scg5 & Slc39a1 \\
\hline
\end{tabular}

\section{Significant Phosphoproteins}

Ankrd33b

Zfp41

Hist1h3b

Atp1a2

.

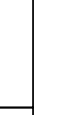


Figure 9
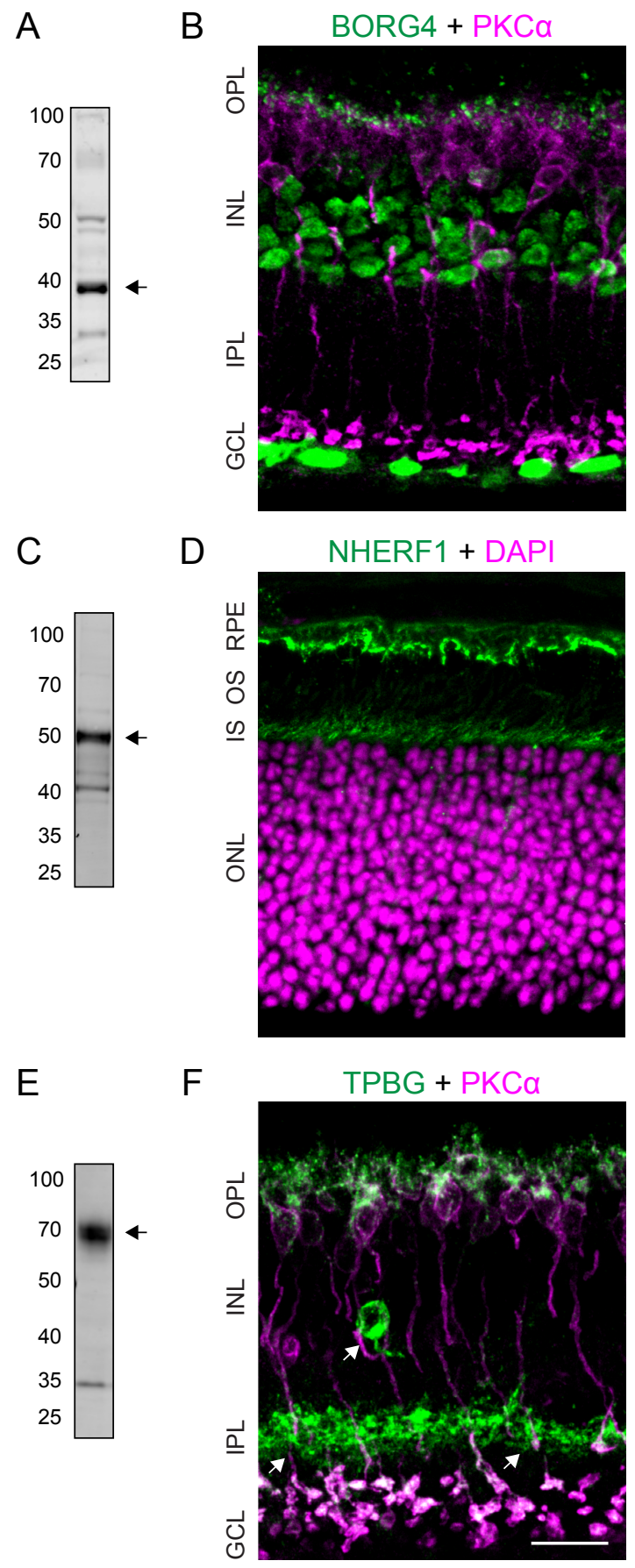